\title{
A study of truncation effects in boundary flows of the Ising model on a strip
}

\author{
G. Zs. Tóth \\ Theoretical Physics Research Group of the Hungarian Academy of Sciences \\ at Eötvös University, 1117 Budapest, Pázmány Péter Sétány 1/A, Hungary \\ E-mail: tgzs@ludens.elte.hu
}

23 April 2007

Journal reference: J. Stat. Mech. (2007) P04005

\begin{abstract}
We investigate the idea that the effect of the truncation applied in the TCSA method on the spectrum coincides with the effect of a suitable changing of the coefficients of the terms in the Hamiltonian operator. The investigation is done in the case of the critical Ising model on a strip with an external magnetic field on one of the boundaries. A detailed quantum field theoretical description of this model is also given, and we propose a description as a perturbation of the infinite coupling limit. The investigation is also carried out for a truncation method which preserves the solvability of the model. The results of perturbative and numerical calculations presented support the above idea and show that the qualitative behaviour of the truncated spectrum as a function of the coupling constant depends on the truncation method.
\end{abstract}

Keywords: integrable quantum field theory, conformal field theory, finite-size scaling, renormalization group 


\section{Contents}

1 Introduction

2 The exact spectrum 6

2.1 The free model . . . . . . . . . . . . . . . . . . . . . . . 7

2.2 The perturbed model . . . . . . . . . . . . . . . . . . . . . 9

3 Reverse description $\quad 12$

3.1 The free model . . . . . . . . . . . . . . . . . . . . 12

3.2 The perturbed model . . . . . . . . . . . . . . . . . 13

4 Exact spectrum in the Mode Truncated version 14

4.1 The free model . . . . . . . . . . . . . . . . . . 14

4.2 The perturbed model . . . . . . . . . . . . . . . . 15

5 Perturbative results $\quad 17$

5.1 Mode Truncation scheme . . . . . . . . . . . . . . . . 17

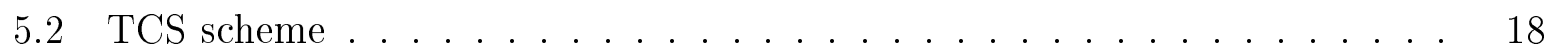

6 Numerical results $\quad 18$

6.1 Mode Truncation Scheme . . . . . . . . . . . . . . . . . . . . . . . 19

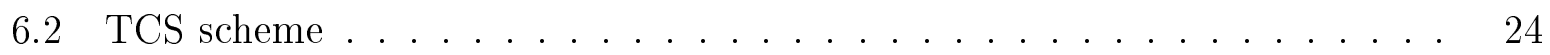

7 Discussion 29

A Distributions on closed line segments 30

B Extension to Section 2 31

C Bethe-Yang equations 35

D Power series expansion of the energy levels 36

\section{Introduction}

The main subject of this paper is an investigation of the TCSA (truncated conformal space approach) method, which is a numerical method introduced in [1] for the calculation of the spectra and eigenvectors of Hamiltonian operators of perturbed conformal field theories in finite volume in two spacetime dimensions. The Hamiltonian operators of these theories have the form $H=H_{0}+H_{I}$, where $H_{0}$ has a known discrete spectrum. The method consists in (numerically) diagonalizing the finite matrix $H^{T C S A}\left(n_{c}\right)=P\left(n_{c}\right)\left(H_{0}+H_{I}\right) P\left(n_{c}\right)$, where 
$P\left(n_{c}\right)$ is the projection on the subspace spanned by the eigenstates of $H_{0}$ belonging to the lowest $n_{c}+1$ energy levels, and $n_{c}$ is the truncation parameter. This gives an approximation to the eigenvalues and eigenvectors of $H$.

The uses of the data obtained by TCSA include the verification of results obtained by other methods, for example in [2], the extraction of resonance widths [3], the mapping of the phase structure of certain quantum field theories as in [4, 5], and the finding of renormalization group flow fixed points as in [6]. Our investigation is related to the use of TCSA for the study of boundary renormalization group flows (i.e. of flows generated by boundary perturbations) between minimal boundary conformal field theories defined on the strip $[0, L] \times \mathbb{R}$. A brief review of the areas where boundary conformal field theory and flows between them play important role can be found in [7].

By boundary flow we mean a one-parameter family of models, the parameter being the width (or 'volume') $L$ of the strip. In the simplest case the parameter can be taken to be a coupling constant $h$ instead of $L$ and the models have the Hamiltonian operators $H=H_{0}+h H_{I} . H_{0}$ is the Hamiltonian operator of a boundary conformal field theory, $h$ is allowed to vary from 0 to $\infty$ or from 0 to $-\infty$. $H_{I}$ is a relevant boundary field taken at a certain initial time.

A particular problem of interest is that of finding values of $h$ other than 0 , called fixed points, where the model corresponding to $H_{0}+h H_{I}$ is a conformal field theory, and identifying these conformal field theories. The TCSA can be used for this purpose if the Hilbert space of the conformal field theory corresponding to $h=0$ consists of finitely many (or countably many) irreducible representations of the Virasoro algebra. Boundary conformal minimal models satisfy this condition. We refer the reader to [8, 9, 10, 11, 6] for boundary flows and the application of the TCSA to study them.

Regarding the spectra at nonzero values of $h$, conformal symmetry can be recognized by the equal distances between neighbouring energy levels; the representation content can be identified by the degeneracy of the energy levels. It should be noted that perturbation theory and other methods can also be used (see e.g. [7, 13, 14, 15, 16, 17]) to explore flows. An important problem regarding the TCSA is that it gives approximate data, and our knowledge of the precise relation between this data and the exact spectrum is still limited (see [18 for already existing results). A good understanding of the effect of the truncation could be used to improve the TCSA data and to explain the qualitative features of TCSA pictures of flows, and generally it would make the results obtained using TCSA data better founded.

An idea proposed by G.M.T. Watts and K. Graham [19, 20, recently is that the effect of the truncation on the spectrum is equivalent to a suitable change of the coefficients of the terms in $H$, i.e. the spectrum of $H^{T C S A}\left(h, n_{c}\right)$ is equal, in an approximation at least, to the spectrum of

$$
H^{r}\left(h, n_{c}\right)=s_{0}\left(h, n_{c}\right) H_{0}+s_{1}\left(h, n_{c}\right) H_{I}+s_{2}\left(h, n_{c}\right) H_{I, 2}+\ldots,
$$

which we shall call renormalized Hamiltonian operator. $s_{0}, s_{1}, \ldots$ are suitable functions 
and $H_{I, 2}, \ldots$ are suitable operators. $H_{I, 2}, \ldots$ should be primary or descendant bulk or boundary operators. Our main purpose in this paper is to investigate the validity of this picture.

We do this investigation in the case of a relatively simple model: the perturbed boundary conformal field theory on the strip $[0, L] \times \mathbb{R}$ with Hamiltonian operator

$$
H=\frac{\pi}{L} L_{0}+h L^{-1 / 2} \phi_{1 / 2}(x=L, t=0) .
$$

The unperturbed ( $h=0$ ) model is the $c=1 / 2$ unitary conformal minimal model, i.e. the continuum limit of the critical Ising model, with the Cardy boundary condition 0 on the left and $1 / 16$ on the right. $\frac{\pi}{L} L_{0}$ is the Hamiltonian operator of this model. $L_{0}$ is the 'zero index' Virasoro generator. $L$ will be kept fixed at the value $L=1$. The Hilbert space of the unperturbed model is the single $c=1 / 2, h=1 / 16$ irreducible highest weight representation of the Virasoro algebra. (Here and later on the coupling constant and the highest weight are both denoted by $h$, but it should be clear from the context which one is meant.) The field $\phi_{1 / 2}(L, t)$ is the weight $1 / 2$ boundary primary field on the right boundary, which is also known in the literature as the boundary spin operator [21, 22, 23. The coupling constant $h$ can also be regarded as a constant external boundary magnetic field, which is coupled to the boundary spin operator. The model (2) is also referred to as the critical Ising model on the strip with boundary magnetic field. The perturbation $h \phi_{1 / 2}(L, t=0)$ violates the conformal symmetry, which is nevertheless restored in the $h \rightarrow \pm \infty$ limit. It is known that in the $h \rightarrow \infty$ limit the $c=1 / 2, h=1 / 2$ representation is realized; in the $h \rightarrow-\infty$ limit the $c=1 / 2, h=0$ representation is realized (see e.g. [13, 14, 23, 24]). This can be written in a shorthand form as

$$
\begin{array}{ll}
(1 / 2,1 / 16)+\phi_{1 / 2} & \rightarrow(1 / 2,1 / 2) \\
(1 / 2,1 / 16)-\phi_{1 / 2} & \rightarrow(1 / 2,0) .
\end{array}
$$

We have chosen the model (2) because it is integrable, furthermore it is relatively easy to handle; in particular the spectrum can be calculated analytically in terms of simple functions and the application of Rayleigh-Schrödinger perturbation theory is also relatively easy. Investigations in other perturbed conformal minimal models, especially in the case of the tricritical Ising model and generally in the case of a perturbation by the field $\phi_{(13)}$, are carried out in [25] (see also [20]).

It should be noted that the Ising model with boundary magnetic field has been studied much, especially on the lattice and on the half-line. We refer the reader to [12, 23, 26, 27] and the references in them for further information.

To recognize conformal symmetry and to identify the representation content it is sufficient to look at the ratios of the energy gaps; therefore one often considers normalized spectra which are obtained by subtracting the ground state energy and dividing by the lowest energy gap. The normalized exact and TCSA spectra for the flows (3), (4) as a function 
of the logarithm of $h$ can be seen in Figure 8. An interesting feature of these TCSA spectra is that they appear to correspond to the flows $(1 / 2,1 / 16) \rightarrow(1 / 2,1 / 2) \rightarrow(1 / 2,1 / 16)$ and $(1 / 2,1 / 16) \rightarrow(1 / 2,0) \rightarrow(1 / 2,1 / 16)$, i.e. second flows appear to be present after the normal flows. Flows in other minimal models also show this behaviour [20, 25]. One application of the picture (1) could be the explanation of this phenomenon.

Following Watts' proposal based on the look of the TCSA spectra shown in Figure 8 we assume that only the first two terms are nonzero in (1):

$$
H^{r}\left(h, n_{c}\right)=s_{0}\left(h, n_{c}\right) H_{0}+s_{1}\left(h, n_{c}\right) H_{I} .
$$

In summary, we look for answers for the following questions: 1 . Does the spectrum of (5) agree with the TCSA spectrum in some approximation with a suitable choice of the functions $s_{0}$ and $s_{1}$ ? 2. How can we explain the 'second' flows in the TCSA spectra?

We determine the exact spectrum of (2) using an essentially known (see e.g. [23, 26, 27, 28, 29, 30]) quantum field theoretic representation of this operator. In this representation the operator (2) is a quadratic expression of fermionic fields. We extract the spectrum from the (quantum) field equations, which are linear.

Concerning the TCSA Hamiltonian operator, the main difficulty we encounter is that it is hard to handle the TCSA spectra analytically even if the non-truncated model is exactly solvable. Therefore, hoping that we can gain some insight by looking at a similar but exactly solvable truncation method, we tried another truncation method which we call mode truncation. The mode truncated model can be solved exactly, but it turns out, rather unexpectedly, that the behaviour of the spectrum for large values of $h$ is different from the behaviour of the TCSA spectrum, namely the qualitative behaviour of the mode truncated spectrum is very similar to that of the exact spectrum; the second flows are not present. This, besides leaving the second question open, raises the problem of finding the possible behaviours for large values of $h$ and their dependence on the truncation method, and whether the mode truncation method can be generalized to other models.

We also apply the Rayleigh-Schrödinger perturbation theory to verify the validity of (5) for both the TCSA and mode truncation methods.

A further calculation that we do is a numerical comparison of the exact and TCSA spectra. We perform the same calculation for the mode truncation scheme as well.

Our secondary aim in this paper, which is partially independent of the problem of the TCSA approximation, is to provide a detailed description of the quantum field theoretic representation of (2). Although this was studied in the literature (e.g. in [28] and especially in [26] (at finite temperature), and on the half-line in several papers), we give a description in which the perturbed Hamiltonian operator structure, on which the TCSA is based, is emphasized and observed consistently. In a calculation using the Bethe-Yang equations (which we also present in the appendix) or in the approach of [26] where boundary conditions play central role, the link with the TCSA formulation would not be entirely obvious. Furthermore, the formulation we present is also suitable for Rayleigh-Schrödinger perturbation theory and for the treatment of the mode truncated version. We do not consider the 
classical level of the field theoretic model, mainly because it is irrelevant to our problem. We remark that the same model with massive unperturbed part (see e.g. [12]) could be studied along the same lines. The field theoretic approach also raises the problem of defining distributions (or similar objects) on a closed interval. We do not know of a systematic exposition of this subject (neither for a closed interval nor for the half-line), although it would be needed for boundary field theory. In this paper we use distributions on closed intervals, restricting to the most necessary formulae only. These formulae are collected in Appendix A.

We remark that our TCSA program relies on the conformal transformation properties only and does not make use of the representation mentioned above.

The contents of the paper are the following:

Section 2 contains the field theoretical description of the model (2), in particular the calculation of the exact spectrum. Further details concerning the boundary conditions, the normalization of interacting creation and annihilation operators, the relation between free and interacting creation and annihilation operators, matrix elements of the interacting fields, identities for the Dirac delta on the strip and the expression of eigenstates in terms of the unperturbed eigenstates are deferred to Appendix B.

In Section 3 we propose a description of (2) as a perturbation of the $h \rightarrow \pm \infty$ limiting case, which cannot be found in the literature. An interesting feature of this description, which we call reverse description, is that the perturbing operator is non-relevant. We calculate the exact spectrum in a similar way as in the case of the standard description mentioned above. This reverse description is motivated by the presence of the second (reverse) flows in the TCSA spectra.

In Section 4 we describe the mode truncated model and calculate its spectrum. This is done along the same lines as in the non-truncated case.

Section 5 contains the perturbative results for the $s_{0}$ and $s_{1}$ functions.

Section [6] contains the results of the numerical test of the approximation by (5) for the TCSA and the mode truncation schemes.

The paper contains further appendices beyond those mentioned above.

In Appendix $\mathrm{C}$ we describe the spectrum using the Bethe-Yang equations. We find that they give the exact result in this case.

Appendix D contains the power series for the exact, TCSA and mode truncated energy levels up to third order in $h$ which we obtained by means of the Rayleigh-Schrödinger perturbation theory.

Conclusions and some directions for future work are given in Section 7 .

\section{The exact spectrum}

In this section we present a quantum field theoretic description of the model (2) and calculate its energy spectrum. 


\subsection{The free model}

The defining constituents of the unperturbed model are the following: two fermion fields $\Psi_{1}(x, t)$ and $\Psi_{2}(x, t)$ and a fermionic operator $A_{2}(t)$ with the anticommutators

$$
\begin{aligned}
\left\{\Psi_{1}(x, t), \Psi_{1}(y, t)\right\} & =\left\{\Psi_{2}(x, t), \Psi_{2}(y, t)\right\}=4 L \delta(x-y) \\
\left\{\Psi_{1}(x, t), \Psi_{2}(y, t)\right\} & =-4 L[\delta(x+y)+\delta(x+y-2 L)] \\
\left\{A_{2}(t), \Psi_{1}(x, t)\right\} & =\left\{A_{2}(t), \Psi_{2}(x, t)\right\}=0 \\
\left\{A_{2}(t), A_{2}(t)\right\} & =2
\end{aligned}
$$

and the relations

$$
\Psi_{1}(x, t)^{\dagger}=\Psi_{1}(x, t) \quad \Psi_{2}(x, t)^{\dagger}=\Psi_{2}(x, t) \quad A_{2}(t)^{\dagger}=A_{2}(t),
$$

the Hamiltonian operator

$$
H_{0}=-\frac{\mathrm{i}}{8 L} \int_{0}^{L} \mathrm{~d} x \Psi_{1}(x, 0) \partial_{x} \Psi_{1}(x, 0)+\frac{\mathrm{i}}{8 L} \int_{0}^{L} \mathrm{~d} x \Psi_{2}(x, 0) \partial_{x} \Psi_{2}(x, 0),
$$

the equations of motion

$$
\begin{aligned}
\frac{\mathrm{d}}{\mathrm{d} t} A_{2}(t)= & {\left[\mathrm{i} H_{0}, A_{2}(t)\right]=0 } \\
\partial_{t} \Psi_{1}(x, t)= & {\left[\mathrm{i} H_{0}, \Psi_{1}(x, t)\right] } \\
= & -\partial_{x} \Psi_{1}(x, t)+\frac{1}{2}[\Theta(-x)+\Theta(x-L)]\left[-\partial_{x} \Psi_{2}(x, t)+\partial_{x} \Psi_{1}(x, t)\right] \\
& +\frac{1}{2}\left[-\Psi_{1}(0, t)-\Psi_{2}(0, t)\right] \delta(x)+\frac{1}{2}\left[\Psi_{1}(L, t)+\Psi_{2}(L, t)\right] \delta(x-L), \\
\partial_{t} \Psi_{2}(x, t)= & {\left[\mathrm{i} H_{0}, \Psi_{2}(x, t)\right] } \\
= & \partial_{x} \Psi_{2}(x, t)+\frac{1}{2}[\Theta(-x)+\Theta(x-L)]\left[-\partial_{x} \Psi_{2}(x, t)+\partial_{x} \Psi_{1}(x, t)\right] \\
& +\frac{1}{2}\left[\Psi_{1}(0, t)+\Psi_{2}(0, t)\right] \delta(x)+\frac{1}{2}\left[-\Psi_{1}(L, t)-\Psi_{2}(L, t)\right] \delta(x-L) .
\end{aligned}
$$

The fermion fields, which are one-component real fermion fields with zero mass, have the following expansion:

$$
\begin{aligned}
& \Psi_{1}(x, t)=\sum_{k \in \frac{\pi}{L} \mathbb{Z}, k>0} \sqrt{2}\left[a(k) \mathrm{e}^{\mathrm{i} k(t-x)}+a^{\dagger}(k) \mathrm{e}^{-\mathrm{i} k(t-x)}\right]+A_{1} \\
& \Psi_{2}(x, t)=\sum_{k \in \frac{\pi}{L} \mathbb{Z}, k>0} \sqrt{2}\left[-a(k) \mathrm{e}^{\mathrm{i} k(t+x)}-a^{\dagger}(k) \mathrm{e}^{-\mathrm{i} k(t+x)}\right]-A_{1}
\end{aligned}
$$




$$
\begin{aligned}
a(k)^{\dagger} & =a(-k) \\
\left\{a\left(k_{1}\right), a\left(k_{2}\right)\right\} & =\delta_{k_{1},-k_{2}} \\
\left\{A_{2}, A_{1}\right\} & =0 \\
\left\{A_{2}, a(k)\right\} & =0 .
\end{aligned}
$$

The fermion fields and $A_{2}$ are dimensionless.

A unitary representation for the above operator algebra is defined by the following formulae: an orthonormal basis of the Hilbert space is

$$
a\left(k_{1}\right) a\left(k_{2}\right) a\left(k_{3}\right) \ldots a\left(k_{n}\right)|u\rangle \quad a\left(k_{1}\right) a\left(k_{2}\right) a\left(k_{3}\right) \ldots a\left(k_{n}\right)|v\rangle,
$$

where $k_{1}>k_{2}>k_{3}>\cdots>k_{n}>0, k_{i} \in \pi \mathbb{Z} / L$ and $n \geq 0$,

$$
\begin{aligned}
& A_{1} a\left(k_{1}\right) a\left(k_{2}\right) a\left(k_{3}\right) \ldots a\left(k_{n}\right)|u\rangle=(-1)^{n} a\left(k_{1}\right) a\left(k_{2}\right) a\left(k_{3}\right) \ldots a\left(k_{n}\right)|v\rangle \\
& A_{1} a\left(k_{1}\right) a\left(k_{2}\right) a\left(k_{3}\right) \ldots a\left(k_{n}\right)|v\rangle=(-1)^{n} a\left(k_{1}\right) a\left(k_{2}\right) a\left(k_{3}\right) \ldots a\left(k_{n}\right)|u\rangle \\
& A_{2} a\left(k_{1}\right) a\left(k_{2}\right) a\left(k_{3}\right) \ldots a\left(k_{n}\right)|u\rangle=(-1)^{n}(-\mathrm{i}) a\left(k_{1}\right) a\left(k_{2}\right) a\left(k_{3}\right) \ldots a\left(k_{n}\right)|v\rangle \\
& A_{2} a\left(k_{1}\right) a\left(k_{2}\right) a\left(k_{3}\right) \ldots a\left(k_{n}\right)|v\rangle=(-1)^{n}(+\mathrm{i}) a\left(k_{1}\right) a\left(k_{2}\right) a\left(k_{3}\right) \ldots a\left(k_{n}\right)|u\rangle .
\end{aligned}
$$

The condition $k_{1}>k_{2}>k_{3}>\cdots>k_{n}>0, k_{i} \in \pi \mathbb{Z} / L$ and $n \geq 0$ also applies below if not stated otherwise. $a(k)|u\rangle=0, a(k)|v\rangle=0$ if $k<0$. The energy eigenvalue of a basis vector $a\left(k_{1}\right) a\left(k_{2}\right) a\left(k_{3}\right) \ldots a\left(k_{n}\right)|u\rangle$ is $\sum_{i=1}^{n} k_{i}$, and the same applies if $u$ is replaced by $v$.

The following boundary conditions are satisfied by the fermion fields:

$$
\left\langle E_{1}\left|\Psi_{1}(0, t)+\Psi_{2}(0, t)\right| E_{2}\right\rangle=0 \quad\left\langle E_{1}\left|\Psi_{1}(L, t)+\Psi_{2}(L, t)\right| E_{2}\right\rangle=0,
$$

where $\left|E_{1}\right\rangle$ and $\left|E_{2}\right\rangle$ are arbitrary energy eigenstates.

A representation of the Virasoro algebra can be defined on the Hilbert space in the following way (which is well known essentially in conformal field theory; see [31, 32]):

$$
\begin{aligned}
L_{N} & =\frac{L}{2 \pi} \sum_{k \in \frac{\pi}{L} \mathbb{Z}}-k a(-k) a\left(k-\frac{N \pi}{L}\right) \\
L_{0} & =\frac{L}{2 \pi} \sum_{k \in \frac{\pi}{L} \mathbb{Z}}[-k: a(-k) a(k):]+\frac{1}{16},
\end{aligned}
$$

where $N= \pm 1, \pm 2, \ldots, a(0)=(1 / \sqrt{2}) A_{1},::$ denotes the normal ordering for fermionic creation and annihilation operators, $: a(-k) a(k):=a(-k) a(k)$ if $k<0,: a(-k) a(k):=$ $-a(k) a(-k)$ if $k>0$. It can be verified that in the above representation of the $a(k)$ and $A_{2}$ operators $L_{N}, N \in \mathbb{Z}$ satisfy the usual relations of the generators of the Virasoro algebra with central charge $c=1 / 2 . A_{2}$ commutes with the $L_{N} .|v\rangle$ and $|u\rangle$ are highest weight states with weight $1 / 16$ and the Hilbert space decomposes into two copies of the 
$M(c=1 / 2, h=1 / 16)$ unitary highest weight representation of the Virasoro algebra. The invariant subspace belonging to $u$ is spanned by the vectors $a\left(k_{1}\right) a\left(k_{2}\right) \ldots a\left(k_{n}\right)|u\rangle$ with $n$ even and $a\left(k_{1}\right) a\left(k_{2}\right) \ldots a\left(k_{n}\right)|v\rangle$ with $n$ odd. The invariant subspace belonging to $v$ is spanned by the vectors $a\left(k_{1}\right) a\left(k_{2}\right) \ldots a\left(k_{n}\right)|u\rangle$ with $n$ odd and $a\left(k_{1}\right) a\left(k_{2}\right) \ldots a\left(k_{n}\right)|v\rangle$ with $n$ even. These subspaces will be called $u$ and $v$ sectors. The relation between $H_{0}$ and $L_{0}$ is

$$
H_{0}=\frac{\pi}{L} L_{0}-\frac{1}{16} \frac{\pi}{L}
$$

The fields $\Psi_{1}$ and $\Psi_{2}$ can be written as $\Psi_{1}(z)=\sqrt{2} \sum_{n \in \mathbb{Z}} \tilde{a}(n) z^{n}, \Psi_{2}(\bar{z})=\sqrt{2} \sum_{n \in \mathbb{Z}}-\tilde{a}(n) \bar{z}^{n}$, where $\tilde{a}(n)=a(k), k=n \pi / L, z=\exp \left[\mathrm{i} \frac{\pi}{L}(t-x)\right], \bar{z}=\exp \left[\mathrm{i} \frac{\pi}{L}(t+x)\right]$. For $\epsilon(z)=\Psi_{1}(z) / \sqrt{z}$ and $\bar{\epsilon}(\bar{z})=\Psi_{2}(\bar{z}) / \sqrt{\bar{z}}$ we have $\left[L_{N}, \epsilon(z)\right]=z^{N+1} \frac{\mathrm{d} \epsilon}{\mathrm{d} z}(z)+\frac{1}{2}(N+1) z^{N} \epsilon(z)$ and $\left[L_{N}, \bar{\epsilon}(\bar{z})\right]=$ $\bar{z}^{N+1} \frac{\mathrm{d} \bar{\epsilon}}{\mathrm{d} \bar{z}}(\bar{z})+\frac{1}{2}(N+1) \bar{z}^{N} \bar{\epsilon}(\bar{z})$, i.e. $\epsilon(z)$ and $\bar{\epsilon}(\bar{z})$ are chiral Virasoro primary fields of weight 1/2. The same relations apply to the fields $A_{2} \epsilon$ and $A_{2} \bar{\epsilon}$, in particular $A_{2} \epsilon$ and $A_{2} \bar{\epsilon}$ are also chiral primary fields of weight $1 / 2 . A_{2} \epsilon$ and $A_{2} \bar{\epsilon}$ have zero matrix elements between the $u$ and $v$ sector, whereas $\epsilon(z)$ and $\bar{\epsilon}(\bar{z})$ have zero matrix elements within the $u$ and $v$ sectors. In the above equations the domains of $z$ and $\bar{z}$ are extended to the whole complex plane.

The operator $A_{2}$ is an auxiliary operator and if it is omitted, then it is possible to represent the fields so that the Hilbert space is a single Virasoro module $(1 / 2,1 / 16)$. It is the next section where the presence of $A_{2}$ will be really useful.

\subsection{The perturbed model}

The Hamiltonian operator is

$$
\begin{aligned}
H=-\frac{\mathrm{i}}{8 L} \int_{0}^{L} \Psi_{1}(x, 0) \partial_{x} \Psi_{1}(x, 0) \mathrm{d} x+\frac{\mathrm{i}}{8 L} \int_{0}^{L} & \Psi_{2}(x, 0) \partial_{x} \Psi_{2}(x, 0) \mathrm{d} x \\
& +h \mathrm{i} A_{2}(0)\left[\Psi_{2}(L, 0)-\Psi_{1}(L, 0)\right],
\end{aligned}
$$

where $h$ is a coupling constant of dimension mass. The perturbing term

$$
h H_{I}=h \mathrm{i} A_{2}(0)\left[\Psi_{2}(L, 0)-\Psi_{1}(L, 0)\right]
$$

has zero matrix elements between vectors belonging to different sectors, which means that $H$ can be restricted to the $u$ and $v$ sectors separately. These restrictions are denoted by $\left.H\right|_{u}$ and $\left.H\right|_{v}$. $H_{I}$ is also a primary boundary field of weight $1 / 2$ with respect to the Virasoro algebra representation defined in the previous section taken at $t=0$. The matrix elements of $H_{I}$, i.e. of $\left.H_{I}\right|_{u}$ and $\left.H_{I}\right|_{v}$, are uniquely determined by this property and by the values of the matrix elements $\left\langle u\left|H_{I}\right| u\right\rangle$ and $\left\langle v\left|H_{I}\right| v\right\rangle$. It is easy to verify that $2=\left\langle u\left|H_{I}\right| u\right\rangle=-\left\langle v\left|H_{I}\right| v\right\rangle$, and there exists an intertwiner $Y$ of the Virasoro algebra representations on the $u$ and $v$ sectors so that $Y u=v,\left.Y H_{0}\right|_{u} Y^{-1}=\left.H_{0}\right|_{v}$. This also implies that $\left.Y H_{I}\right|_{u} Y^{-1}=-\left.H_{I}\right|_{v}$. This means finally that we can restrict to $0 \leq h \leq \infty$, 
and the $u$ sector and $\left.H\right|_{u}$ will correspond to the $h \geq 0$ case of (2) and to (3), the $v$ sector and $\left.H\right|_{v}$ will correspond to the $h \leq 0$ case of (2) and to (44). For $0 \leq h \leq \infty$ the operator (30) describes in the two sectors the two flows mentioned in the Introduction. The precise relation between the $h$ in (2) in the Introduction and the $h$ in (30) is the following: $h_{\text {Introd }}=2 L^{1 / 2} h$ in the $u$ sector, $h_{\text {Introd }}=-2 L^{1 / 2} h$ in the $v$ sector. Further on we shall assume that $0<h<\infty$.

The eigenvalues of the Hamiltonian operator (30) are ultraviolet divergent in perturbation theory; the divergence can be removed by adding a term $c h^{2} I$ with appropriate value of the logarithmically divergent (as a function of the cutoff energy) coefficient $c$. This means that the differences of the eigenvalues of $H$ are not ultraviolet divergent. We shall assume that the ground state energy is set to zero, and the $c h^{2} I$ term will not be written explicitly.

The equations of motion are obtained by adding the following terms to the right-hand side of the unperturbed equations of motion (12), (13), (14):

$$
\begin{aligned}
{\left[\mathrm{i} h H_{I}, \Psi_{1}(x, t)\right] } & =8 L h A_{2}(t) \delta(x-L) \\
{\left[\mathrm{i} h H_{I}, \Psi_{2}(x, t)\right] } & =-8 L h A_{2}(t) \delta(x-L) \\
{\left[\mathrm{i} h H_{I}, A_{2}(t)\right] } & =2 h\left(\Psi_{2}(L, t)-\Psi_{1}(L, t)\right) .
\end{aligned}
$$

The initial condition for the fields $\Psi_{1}(x, t), \Psi_{1}(x, t)$ and $A_{2}(t)$ is that at $t=0$ they are equal to the unperturbed fields (of the previous section). The above terms are linear in the fermion fields and $A_{2}$, so the equations of motion for the perturbed theory are linear and by sandwiching these equations between energy eigenstates we get a system of three first-order differential equations for the expectation values of the fields and $A_{2}$. These expectation values can be assumed to take the form

$$
\begin{aligned}
\left\langle E_{1}\left|\Psi_{1}(x, t)\right| E_{2}\right\rangle & =-\mathrm{e}^{\mathrm{i} k(t-x)}-\Theta(x-L) C_{1}(k) \mathrm{e}^{\mathrm{i} k t} \\
\left\langle E_{1}\left|\Psi_{2}(x, t)\right| E_{2}\right\rangle & =\mathrm{e}^{\mathrm{i} k(t+x)}-\Theta(x-L) C_{2}(k) \mathrm{e}^{\mathrm{i} k t} \\
\left\langle E_{1}\left|A_{2}(t)\right| E_{2}\right\rangle & =C_{3}(k) \mathrm{e}^{\mathrm{i} k t},
\end{aligned}
$$

where $C_{1}(k), C_{2}(k), C_{3}(k)$ are finite constants, $\left|E_{1}\right\rangle$ and $\left|E_{2}\right\rangle$ are eigenstates of $H$ and $k=E_{1}-E_{2}$. $\left|E_{1}\right\rangle$ and $\left|E_{2}\right\rangle$ are not necessarily normalized to 1 here. Substituting (35)(37) into the equations of motion we get algebraic equations for $C_{1}(k), C_{2}(k), C_{3}(k)$, which have the following solution:

$$
\begin{gathered}
k L \tan (k L)=16 L^{2} h^{2} \\
\psi_{1}(k)(x, t)=\left\langle E_{1}\left|\Psi_{1}(x, t)\right| E_{2}\right\rangle=-\mathrm{e}^{\mathrm{i} k(t-x)}-\Theta(x-L) \mathrm{i} \sin (k L) \mathrm{e}^{\mathrm{i} k t} \\
\psi_{2}(k)(x, t)=\left\langle E_{1}\left|\Psi_{2}(x, t)\right| E_{2}\right\rangle=\mathrm{e}^{\mathrm{i} k(t+x)}-\Theta(x-L) \mathrm{i} \sin (k L) \mathrm{e}^{\mathrm{i} k t} \\
a_{2}(k)(t)=\left\langle E_{1}\left|A_{2}(t)\right| E_{2}\right\rangle=-\frac{\mathrm{i} \sin (k L)}{4 L h} \mathrm{e}^{\mathrm{i} k t} .
\end{gathered}
$$


Equation (38) is the formula that determines the possible values of $k$ at a given value of $h$ and $L$ and so the spectrum of $H$ up to an undetermined additive overall constant.

The assumption (35), (36) is motivated by the fact that the equations of motion for $x \in(0, L)$ are $\left(\partial_{t}+\partial_{x}\right) \Psi_{1}(x, t)=0,\left(\partial_{t}-\partial_{x}\right) \Psi_{2}(x, t)=0$. We remark that the equations of motion could also be solved directly without making any assumptions on the form of the expectation values.

Introducing the notation

$$
n(k)=\left(\psi_{1}(k), \psi_{2}(k), a_{2}(k)\right)
$$

the mode expansion of $\left(\Psi_{1}, \Psi_{2}, A_{2}\right)$ is

$$
\left(\Psi_{1}, \Psi_{2}, A_{2}\right)=\sum_{k \in S} b(k) n(k)
$$

where the $b(k)$ are creation/annihilation operators and the summation is done over the set $S$ of all real solutions of (38). The $b(k)$ satisfy the relations (see Appendix B for details)

$$
\left\{b\left(k_{1}\right), b\left(k_{2}\right)\right\}=\delta_{k_{1}+k_{2}, 0} \frac{4 L k_{1}}{2 L k_{1}+\sin \left(2 L k_{1}\right)}
$$

and $b(k)^{\dagger}=b(-k)$.

The Hilbert space is spanned by the orthogonal eigenstates $b\left(k_{1}\right) b\left(k_{2}\right) b\left(k_{3}\right) \ldots b\left(k_{n}\right)\left|0_{h}\right\rangle$ where $k_{1}>k_{2}>\cdots>k_{n}>0,\left|0_{h}\right\rangle$ is the ground state, which is unique, and $b(k)\left|0_{h}\right\rangle=0$ if $k<0$. The eigenvalues of these states are $\sum_{i=1}^{n} k_{i}$. The eigenvector $b\left(k_{1}\right) b\left(k_{2}\right) \ldots b\left(k_{n}\right)\left|0_{h}\right\rangle$ belongs to the $v$ sector if $n$ is even and to the $u$ sector if $n$ is odd. The first few energy gaps (i.e. energies relative to the lowest energy) within the two sectors are shown in Figure 7 as functions of $\ln (h)$ with $L=1$.

In the $h: 0 \rightarrow \infty$ limit

$$
k(n, h) \rightarrow k(n, 0)+\frac{1}{2} \frac{\pi}{L}=\frac{n \pi}{L}+\frac{1}{2} \frac{\pi}{L},
$$

where $k(n, h)$ is the $n$-th nonnegative root of (38) as a function of $h, k(n, 0)=n \pi / L$, $n=0,1,2, \ldots$; and $\left\{b\left(k_{i}\right), b\left(k_{j}\right)\right\} \rightarrow 2 \delta_{k_{i}+k_{j}, 0}$. It can be verified that in the $h \rightarrow \infty$ limit the $(c=1 / 2, h=0)$ representation of the Virasoro algebra can be introduced in the $v$ sector and the $(c=1 / 2, h=1 / 2)$ representation can be introduced in the $u$ sector. One can write expressions (which are well known essentially, see [31, 32]) for the generators in terms of the $b(k)$ similar to (27), (28). Therefore $h: 0 \rightarrow \infty$ corresponds to the $(1 / 2,1 / 16) \rightarrow(1 / 2,0)$ flow in the $v$ sector and to the $(1 / 2,1 / 16) \rightarrow(1 / 2,1 / 2)$ flow in the $u$ sector. We remark that the easiest way to determine which representations are realized in the $h \rightarrow \infty$ limit is by counting the degeneracies of the first few energy levels (separately in the two sectors). 


\section{Reverse description}

In this section we propose the description of the model (30) as a perturbation of its $h \rightarrow \infty$ limit. This is motivated by the presence of the second (reverse) flows in the TCSA spectra (Figure (8) ). It should be noted that the meaning of the notation $H_{0}$ or $H_{I}$ etc differs from that in Section 2. The precise correspondence between the quantities in this section and in the previous section will be given explicitly for the coupling constant and for the spectrum.

\subsection{The free model}

The fundamental objects of the model at $h=\infty$ are two one-component real massless fermion fields $\Phi_{1}(x, t), \Phi_{2}(x, t)$ with the anticommutation relations

$$
\begin{aligned}
& \left\{\Phi_{1}(x, t), \Phi_{1}(y, t)\right\}=\left\{\Phi_{2}(x, t), \Phi_{2}(y, t)\right\}=4 L \delta(x-y) \\
& \left\{\Phi_{1}(x, t), \Phi_{2}(y, t)\right\}=-4 L[\delta(x+y)-\delta(x+y-2 L)]
\end{aligned}
$$

and reality property $\Phi_{1}(x, t)^{\dagger}=\Phi_{1}(x, t), \Phi_{2}(x, t)^{\dagger}=\Phi_{2}(x, t) . \Phi_{1}$ and $\Phi_{2}$ are dimensionless. The Hamiltonian operator is

$$
H_{0}=-\frac{\mathrm{i}}{8 L} \int_{0}^{L} \mathrm{~d} x \Phi_{1}(x, 0) \partial_{x} \Phi_{1}(x, 0)+\frac{\mathrm{i}}{8 L} \int_{0}^{L} \mathrm{~d} x \Phi_{2}(x, 0) \partial_{x} \Phi_{2}(x, 0) .
$$

The equations of motion are

$$
\begin{aligned}
\partial_{t} \Phi_{1}(x, t)= & {\left[\mathrm{i} H_{0}, \Phi_{1}(x, t)\right]=-\partial_{x} \Phi_{1}(x, t) } \\
& +\frac{1}{2} \delta(x-L)\left[\Phi_{1}(L, t)-\Phi_{2}(L, t)\right]+\frac{1}{2} \delta(x)\left[-\Phi_{1}(0, t)-\Phi_{2}(0, t)\right] \\
& +\frac{1}{2} \Theta(-x)\left[\partial_{x} \Phi_{1}(x, t)-\partial_{x} \Phi_{2}(x, t)\right]+\frac{1}{2} \Theta(x-L)\left[\partial_{x} \Phi_{1}(x, t)+\partial_{x} \Phi_{2}(x, t)\right] \\
\partial_{t} \Phi_{2}(x, t)=[ & {\left[\mathrm{i} H_{0}, \Phi_{2}(x, t)\right]=\partial_{x} \Phi_{2}(x, t) } \\
& +\frac{1}{2} \delta(x-L)\left[\Phi_{1}(L, t)-\Phi_{2}(L, t)\right]+\frac{1}{2} \delta(x)\left[\Phi_{1}(0, t)+\Phi_{2}(0, t)\right] \\
+ & \frac{1}{2} \Theta(-x)\left[\partial_{x} \Phi_{1}(x, t)-\partial_{x} \Phi_{2}(x, t)\right]+\frac{1}{2} \Theta(x-L)\left[-\partial_{x} \Phi_{1}(x, t)-\partial_{x} \Phi_{2}(x, t)\right] .
\end{aligned}
$$

The fermion fields have the following mode expansion:

$$
\begin{aligned}
& \Phi_{1}(x, t)=\sum_{k \in \frac{\pi}{L} \mathbb{Z}+\frac{\pi}{2 L}} \sqrt{2} a(k) \mathrm{e}^{\mathrm{i} k(t-x)} \\
& \Phi_{2}(x, t)=\sum_{k \in \frac{\pi}{L} \mathbb{Z}+\frac{\pi}{2 L}}-\sqrt{2} a(k) \mathrm{e}^{\mathrm{i} k(t+x)}
\end{aligned}
$$




$$
\left\{a\left(k_{1}\right), a^{\dagger}\left(k_{2}\right)\right\}=\delta_{k_{1}, k_{2}} \quad a(k)^{\dagger}=a(-k) .
$$

An orthonormal basis for the Hilbert space is formed by the vectors $a\left(k_{1}\right) a\left(k_{2}\right) \ldots a\left(k_{n}\right)|0\rangle$, where $k_{i}>0, k_{i} \in \pi \mathbb{Z} / L+\pi /(2 L), n \geq 0 . a(k)|0\rangle=0$ if $k<0$. The eigenvalue of the eigenvector $a\left(k_{1}\right) a\left(k_{2}\right) \ldots a\left(k_{n}\right)|0\rangle$ is $\sum_{i=1}^{n} k_{i}$. The fermion fields satisfy the following boundary conditions:

$$
\left\langle E_{1}\left|\Phi_{1}(0, t)+\Phi_{2}(0, t)\right| E_{2}\right\rangle=0 \quad\left\langle E_{1}\left|\Phi_{1}(L, t)-\Phi_{2}(L, t)\right| E_{2}\right\rangle=0,
$$

where $\left|E_{1}\right\rangle,\left|E_{2}\right\rangle$ are eigenstates of $H_{0}$.

One can define a representation of the Virasoro algebra on the Hilbert space in the same way as in Section 2.1 (see also [31, 32]). This representation is $(1 / 2,0) \oplus(1 / 2,1 / 2)$. The fields $\Phi_{1}(x, t)$ and $\Phi_{2}(x, t)$ can be converted to weight $1 / 2$ primary fields by multiplying them by a suitable simple exponential factor.

\subsection{The perturbed model}

The perturbed Hamilton operator is

$$
\begin{aligned}
H=-\frac{\mathrm{i}}{8 L} \int_{0}^{L} \mathrm{~d} x \Phi_{1}(x, 0) \partial_{x} \Phi_{1}(x, 0) & +\frac{\mathrm{i}}{8 L} \int_{0}^{L} \mathrm{~d} x \Phi_{2}(x, 0) \partial_{x} \Phi_{2}(x, 0) \\
& +g\left[\mathrm{i}\left(\Phi_{1}+\Phi_{2}\right)(L, 0) \lim _{x \rightarrow L} \partial_{x}\left(\Phi_{2}-\Phi_{1}\right)(x, 0)\right],
\end{aligned}
$$

where $g$ is a dimensionless coupling constant. In the same way as in Section 2.2, the perturbing term has zero matrix elements between vectors belonging to different irreducible representations.

The limit prescription in the perturbing term is important, and the limit $\lim _{x \rightarrow L}$ should be taken at the end of any calculation. It should be assumed that $\lim _{x \rightarrow L} \delta(x-L)=0$, for example, and similarly for the derivatives of $\delta(x-L)$.

It should be noted that the only primary field in the 0 or in the $1 / 2$ representation is the identity operator, all other fields are descendant and non-relevant fields.

The equations of motion are obtained by adding the following terms to the right-hand side of the unperturbed equations of motion (49), (50):

$$
\begin{aligned}
& {\left[\mathrm{i} g H_{I}, \Phi_{1}(x, t)\right]=g 8 L \delta(x-L) \lim _{x \rightarrow L} \partial_{x}\left(\Phi_{2}-\Phi_{1}\right)(x, t)} \\
& {\left[\mathrm{i} g H_{I}, \Phi_{2}(x, t)\right]=g 8 L \delta(x-L) \lim _{x \rightarrow L} \partial_{x}\left(\Phi_{2}-\Phi_{1}\right)(x, t)}
\end{aligned}
$$

where

$$
H_{I}=\left[\mathrm{i}\left(\Phi_{1}+\Phi_{2}\right)(L, 0) \lim _{x \rightarrow L} \partial_{x}\left(\Phi_{2}-\Phi_{1}\right)(x, 0)\right]
$$


Similar steps to those in Section 2.2 can now be taken to obtain $\left\langle E_{1}\left|\Phi_{1}(x, t)\right| E_{2}\right\rangle$ and $\left\langle E_{1}\left|\Phi_{2}(x, t)\right| E_{2}\right\rangle$. In the same way as in Section 2.2, the forms

$$
\begin{aligned}
& \left\langle E_{1}\left|\Phi_{1}(x, t)\right| E_{2}\right\rangle=\mathrm{e}^{\mathrm{i} k(t-x)}+\Theta(x-L) D_{1}(k) \mathrm{e}^{\mathrm{i} k t} \\
& \left\langle E_{1}\left|\Phi_{2}(x, t)\right| E_{2}\right\rangle=-\mathrm{e}^{\mathrm{i} k(t+x)}+\Theta(x-L) D_{2}(k) \mathrm{e}^{\mathrm{i} k t}
\end{aligned}
$$

can be assumed, where $D_{1}(k)$ and $D_{2}(k)$ are finite constants, $\left|E_{1}\right\rangle$ and $\left|E_{2}\right\rangle$ are eigenstates of $H$ and $k=E_{1}-E_{2}$.

Solving the equations of motion for $D_{1}(k), D_{2}(k)$ we get $D_{2}(k)=-D_{1}(k)=\cos (k L)$ and

$$
(k L) \tan (k L)=\frac{-1}{16 g} .
$$

Equation (62) is the formula that determines the spectrum of $H$ up to an overall additive constant. The eigenvalues of $H$ are $k_{1}+k_{2}+\cdots+k_{n}$, where $n \geq 0, k_{i} \geq 0, k_{i} \neq k_{j}$ if $i \neq j$, the $k_{i}$ are real roots of (62) and the lowest eigenvalue is assumed to be set to zero by adding a constant which is not written explicitly. The substitution

$$
g=\frac{-1}{256 L^{2} h^{2}}
$$

converts (62) into (38). Thus $g: 0 \rightarrow-\infty$ corresponds to the flow $(0 \rightarrow 1 / 16) \oplus(1 / 2 \rightarrow$ $1 / 16)$.

In perturbation theory there are divergences if we take $x=L$ in $H_{I}$ at the beginning. However, if one allows $x$ to take general values, then in Rayleigh-Schrödinger perturbation theory for the differences of the energy levels one can expect to get sums at any fixed order which are possible to evaluate. We expect that the evaluation yields, besides non-singular parts, step functions, $\delta(x-L)$ and its derivatives, and the result remains finite after the $x \rightarrow L$ limit.

\section{Exact spectrum in the Mode Truncated version}

\subsection{The free model}

Let $n_{c}$, called the truncation level, be a positive integer. The mode truncated version of the free model described in Section 2.1 is the following:

$$
\begin{aligned}
& \left\{\Psi_{1}(x, t), \Psi_{1}(y, t)\right\}=2\left[1+2 \sum_{k \in \frac{\pi}{L}\left\{1 \ldots n_{c}\right\}} \cos (k(x-y))\right] \\
& \left\{\Psi_{2}(x, t), \Psi_{2}(y, t)\right\}=2\left[1+2 \sum_{k \in \frac{\pi}{L}\left\{1 \ldots n_{c}\right\}} \cos (k(x-y))\right] \\
& \left\{\Psi_{1}(x, t), \Psi_{2}(y, t)\right\}=-2\left[1+2 \sum_{k \in \frac{\pi}{L}\left\{1 \ldots n_{c}\right\}} \cos (k(x+y))\right],
\end{aligned}
$$




$$
\begin{aligned}
& \Psi_{1}(x, t)=\sum_{k \in \frac{\pi}{L}\left\{1 \ldots n_{c}\right\}} \sqrt{2}\left[a(k) \mathrm{e}^{\mathrm{i} k(t-x)}+a^{+}(k) \mathrm{e}^{-\mathrm{i} k(t-x)}\right]+A_{1} \\
& \Psi_{2}(x, t)=\sum_{k \in \frac{\pi}{L}\left\{1 \ldots n_{c}\right\}} \sqrt{2}\left[-a(k) \mathrm{e}^{\mathrm{i} k(t+x)}-a^{+}(k) \mathrm{e}^{-\mathrm{i} k(t+x)}\right]-A_{1} .
\end{aligned}
$$

Equations (17)-(20) apply unchanged. The Hamiltonian operator is

$$
H_{0}=\sum_{k \in \frac{\pi}{L}\left\{1 \ldots n_{c}\right\}} k\left[a(k) a^{+}(k)\right] .
$$

The fields satisfy the following equations of motion:

$$
\begin{aligned}
\partial_{t} \Psi_{1}(x, t) & =\left[\mathrm{i} H_{0}, \Psi_{1}(x, t)\right]=-\partial_{x} \Psi_{1}(x, t) \\
\partial_{t} \Psi_{2}(x, t) & =\left[\mathrm{i} H_{0}, \Psi_{2}(x, t)\right]=\partial_{x} \Psi_{2}(x, t) \\
\frac{\mathrm{d}}{\mathrm{d} t} A_{2}(t) & =\left[\mathrm{i} H_{0}, A_{2}(t)\right]=0 .
\end{aligned}
$$

The Hilbert space and the energy eigenstates are similar to those in Section 2.1, but

$$
k \in \frac{\pi}{L}\left\{-n_{c},-n_{c}+1, \ldots, n_{c}-1, n_{c}\right\}
$$

applies instead of $k \in \pi \mathbb{Z} / L$. The Hilbert space is $2 \times 2^{n_{c}}$ dimensional.

\subsection{The perturbed model}

The perturbed Hamiltonian operator is $H_{0}+h H_{I}$, where

$$
H_{I}=\mathrm{i} A_{2}(0)\left[\Psi_{2}(L, 0)-\Psi_{1}(L, 0)\right] .
$$

The equations of motion are

$$
\begin{aligned}
\partial_{t} \Psi_{1}(x, t) & =\left[\mathrm{i}\left(H_{0}+h H_{I}\right), \Psi_{1}(x, t)\right]=-\partial_{x} \Psi_{1}(x, t)-h A_{2}(t) C_{1}(x) \\
\partial_{t} \Psi_{2}(x, t) & =\left[\mathrm{i}\left(H_{0}+h H_{I}\right), \Psi_{2}(x, t)\right]=\partial_{x} \Psi_{2}(x, t)-h A_{2}(t) C_{2}(x) \\
\frac{\mathrm{d}}{\mathrm{d} t} A_{2}(t) & =\left[\mathrm{i}\left(H_{0}+h H_{I}\right), A_{2}(t)\right]=2 h\left(\Psi_{2}(L, t)-\Psi_{1}(L, t)\right),
\end{aligned}
$$

where

$$
\begin{aligned}
C_{1}(x) & =\left\{\Psi_{1}(x, t), \Psi_{2}(L, t)-\Psi_{1}(L, t)\right\} \\
& =-4\left[1+2 \sum_{k \in \frac{\pi}{L}\left\{1 \ldots n_{c}\right\}} \cos (k(x+L))\right] \\
C_{2}(x) & =\left\{\Psi_{2}(x, t), \Psi_{2}(L, t)-\Psi_{1}(L, t)\right\}=-C_{1}(x) .
\end{aligned}
$$


These equations are linear as in the non-truncated case, so sandwiching them between energy eigenstates gives a system of three first-order linear partial differential equations for the expectation values. The analogue of (38) can be obtained from these equations in the following way: we can eliminate $A_{2}$ :

$$
\begin{aligned}
& \partial_{t}^{2} \Psi_{1}(x, t)=-\partial_{x t} \Psi_{1}(x, t)-2 h^{2}\left(\Psi_{2}(L, t)-\Psi_{1}(L, t)\right) C_{1}(x) \\
& \partial_{t}^{2} \Psi_{2}(x, t)=\partial_{x t} \Psi_{1}(x, t)-2 h^{2}\left(\Psi_{2}(L, t)-\Psi_{1}(L, t)\right) C_{2}(x)
\end{aligned}
$$

and introduce the functions $f_{1}(x), f_{2}(x)$ :

$$
\left\langle E_{1}\left|\Psi_{1}(x, t)\right| E_{2}\right\rangle=f_{1}(x) \mathrm{e}^{\mathrm{i} k t} \quad\left\langle E_{1}\left|\Psi_{2}(x, t)\right| E_{2}\right\rangle=f_{2}(x) \mathrm{e}^{\mathrm{i} k t},
$$

where $k=E_{1}-E_{2}$ and the dependence of $f_{1}$ and $f_{2}$ on $k$ is not denoted explicitly. Sandwiching (80) and (81) between $\left|E_{1}\right\rangle$ and $\left|E_{2}\right\rangle$ gives two first-order inhomogeneous linear ordinary differential equations (with the deviation that the inhomogeneity depends on the unknown functions). We also have the boundary conditions $f_{1}(0)=-f_{2}(0), f_{1}(L)=$ $-f_{2}(L)$. Elementary calculation yields that a solution of the differential equations and the boundary conditions exists if and only if $k$ satisfies the equation

$$
16 h^{2}\left[\frac{1}{k^{2}}+\sum_{k_{0} \in \frac{\pi}{L}\left\{1 \ldots n_{c}\right\}} \frac{2}{k^{2}-k_{0}^{2}}\right]=1,
$$

which is the analogue of (38) and determines the energy of the modes as functions of $h$.

(83) as an algebraic equation for $k$ has finitely many real roots, and if $k$ is a root, then $-k$ is a root as well. All real roots converge to finite values as $h \rightarrow \infty$ except for the pair with the largest absolute value. This pair of roots diverges linearly as $h \rightarrow \infty$. This pair has the largest absolute value already at $h=0$. A consequence of this behaviour is that the lower half of the spectrum, namely those states which do not contain the mode with the highest energy, remains finite as $h \rightarrow \infty$, whereas the higher half of the spectrum, i.e. the states which contain the mode with the highest energy, diverges linearly as $h \rightarrow \infty$ with a common slope. Here it is assumed that the ground state energy is set to zero. In the subsequent sections we shall consider the lower half of the spectrum. The look of this half as a function of $\ln (h)$ is very similar to that of the exact spectrum shown in Figure 7 ,

Applying the formula

$$
1+\sum_{n=1}^{\infty} \frac{2 k^{2}}{k^{2}-4 n^{2} \pi^{2}}=\frac{k / 2}{\tan (k / 2)}
$$

we can easily verify that the limit of (83) as $n_{c} \rightarrow \infty$ is (38). 


\section{$5 \quad$ Perturbative results}

The functions $s_{0}\left(h, n_{c}\right)$ and $s_{1}\left(h, n_{c}\right)$ in $H^{r}=s_{0}\left(h, n_{c}\right) H_{0}+s_{1}\left(h, n_{c}\right) H_{I}$ are determined by the condition that the differences of those eigenvalues of $H^{r}$ that are low compared to the truncation level should be equal to those of the truncated Hamiltonian operator $H^{t}\left(n_{c}\right)$. $H^{t}\left(n_{c}\right)$ is, in particular, the TCSA Hamiltonian operator $H^{T C S A}\left(n_{c}\right)$, or the Hamiltonian operator $H^{M T}\left(n_{c}\right)$ of the mode truncated model. This condition, which we shall call renormalization condition, applies separately and independently within the $u$ and $v$ sectors, and we have in fact a pair $s_{0}^{u}, s_{1}^{u}$ for the $u$ sector and another pair $s_{0}^{v}, s_{1}^{v}$ for the $v$ sector.

The renormalization condition is a very strong condition on $s_{0}$ and $s_{1}$ and generally we cannot expect that it can be satisfied. It is possible, however, that it can be satisfied in certain approximations.

\subsection{Mode Truncation scheme}

Using (156) in Appendix D we can obtain the following results:

The renormalization conditions have a solution if the eigenfunctions are expanded into a power series in $h$ and terms that are higher order than 3 are omitted:

$$
s_{0}\left(h, n_{c}\right)=1+O\left(h^{4}\right) \quad s_{1}\left(h, n_{c}\right)=h+\frac{1}{2}\left(S-S\left(n_{c}\right)\right) L^{2} h^{3}+O\left(h^{4}\right),
$$

where

$$
S=\sum_{n=1}^{\infty} \frac{32}{n^{2} \pi^{2}} \quad S\left(n_{c}\right)=\sum_{n=1}^{n_{c}} \frac{32}{n^{2} \pi^{2}} .
$$

This solution applies to both the $u$ and $v$ sectors and it is exact in $n_{c}$. We remark that

$$
S-S\left(n_{c}\right)=\frac{32}{\pi^{2} n_{c}}+O\left(1 / n_{c}^{2}\right) .
$$

In the MT scheme we can obtain another result that is non-perturbative in $h$ : doing power series expansion in $1 / n_{c}$ we obtain the formula

$$
\frac{1}{k^{2}}+\sum_{n=1}^{n_{c}} \frac{2}{k^{2}-\frac{\pi^{2}}{L^{2}} n^{2}}=\frac{L}{k \tan (k L)}+\frac{2 L^{2}}{\pi^{2}}+\frac{1}{n_{c}}+O\left(1 / n_{c}^{2}\right) .
$$

Omitting the terms which are second or higher order in $1 / n_{c}$, equation (83) takes the form of $(\underline{38})$ if

$$
h_{\mathrm{eff}}=\frac{h}{\sqrt{1-\frac{32 L^{2} h^{2}}{\pi^{2} n_{c}}}}=h+16 h^{3} \frac{L^{2}}{\pi^{2}} \frac{1}{n_{c}}+O\left(1 / n_{c}^{2}\right)
$$

is introduced:

$$
k L \tan (k L)=16 L^{2} h_{\mathrm{eff}}^{2}
$$


This means that rescaling by

$$
s_{0}\left(h, n_{c}\right)=1+O\left(1 / n_{c}^{2}\right) \quad s_{1}\left(h, n_{c}\right)=h+16 h^{3} \frac{L^{2}}{\pi^{2}} \frac{1}{n_{c}}+O\left(1 / n_{c}^{2}\right)
$$

improves the convergence of the mode truncated energy gaps to the exact energy gaps from order $1 / n_{c}$ to order $1 / n_{c}^{2}$ (at least), i.e. the difference between the energy gaps of $s_{0}\left(h, n_{c}\right) H_{0}+s_{1}\left(h, n_{c}\right) H_{I}$ and the energy gaps of $\left(H_{0}+h H_{I}\right)^{M T}$ tends to zero as $1 / n_{c}^{2}$ (at least) for any fixed finite value of $h$, whereas the difference between the energy gaps of $H_{0}+h H_{I}$ and $\left(H_{0}+h H_{I}\right)^{M T}$ tends to zero as $1 / n_{c}$.

\subsection{TCS scheme}

Using (156) in Appendix D we obtain the following results:

The renormalization conditions have a solution if the eigenfunctions are expanded into a power series in $h$ and in $1 / n_{c}$ and terms that are of order higher than 3 in $h$ and 1 in $1 / n_{c}$ are omitted:

$$
\begin{array}{rrr}
s_{0}\left(h, n_{c}\right) & =1+x_{1} h^{2}+y_{1} h^{3}+O\left(h^{4}\right) & \\
s_{1}\left(h, n_{c}\right) & =h+y_{2} h^{2}+x_{2} h^{3}+O\left(h^{4}\right) & \\
x_{1}=\frac{8 L^{2}}{\pi^{2} n_{c}}+O\left(1 / n_{c}^{2}\right) & y_{1}=0+O\left(1 / n_{c}^{2}\right) \\
x_{2}=\frac{1}{2}\left(S-S\left(n_{c}\right)\right) L^{2}+O\left(1 / n_{c}^{2}\right) & y_{2}=0+O\left(1 / n_{c}^{2}\right) .
\end{array}
$$

This solution applies to both sectors. If terms of order $1 / n_{c}^{2}$ are also taken into consideration, then the renormalization conditions do not have a solution. A significant difference between the TCS and mode truncation schemes is that the value of the coefficient $x_{1}$ is zero in the mode truncation scheme but non-zero in the TCS scheme.

\section{$6 \quad$ Numerical results}

In the numerical calculations described in this section the value of $L$ is set equal to 1 . This does not affect the generality of the results. In the calculations we used the same normalizations as in Section 2,

$s_{0}\left(h, n_{c}\right)$ and $s_{1}\left(h, n_{c}\right)$ are calculated from the lowest three energy levels and are given by the following formulae:

$$
\begin{aligned}
& \frac{s_{1}}{s_{0}}(h)=\left(\frac{E_{2}-E_{0}}{E_{1}-E_{0}}\right)^{-1}\left(\left(\frac{E_{2}-E_{0}}{E_{1}-E_{0}}\right)^{t}(h)\right) \\
& s_{0}(h)=\frac{\left(E_{1}-E_{0}\right)^{t}(h)}{\left(E_{1}-E_{0}\right)\left(\frac{s_{1}}{s_{0}}(h)\right)}
\end{aligned}
$$


where the superscriptless quantities are the non-truncated ones and those with the ${ }^{t}$ superscript are obtained by a truncation. $E_{0}, E_{1}, E_{2}$ denote the three lowest energy eigenvalues.

\subsection{Mode Truncation Scheme}

Figure 1 shows the exact and mode truncated spectra as a function of the logarithm of the coupling constant. The truncation level is $n_{c}=9$, and the dimension of the Hilbert space is 512 in each sector. It is remarkable that there is a good qualitative agreement between the mode truncated and exact spectra for all values of $h$.

Figure 2 shows the same spectra, but the lowest gap is normalized to one, i.e. the functions $\frac{E_{i}(h)-E_{0}(h)}{E_{1}(h)-E_{0}(h)}$ are shown. It is remarkable that the agreement between the exact and mode truncated spectra looks considerably better than in the case of not normalized spectra.

Figures 3 .5 show the functions $s_{0}(h), s_{1}(h), s_{1}(h) / s_{0}(h)$ determined by the lowest three energy levels in the $v$ sector via the formulae (96), (97) in various ranges. Figures 4 and 5 also show the curves given by (85) on the left-hand side (red/grey line). $s_{0}(h)$ remains close to 1 and for large values of $h$ it tends to a constant which can be expected to converge to 1 as $n_{c} \rightarrow \infty$. $s_{1}(h)$ also tends to a constant for large values of $h$ which can be expected to increase to infinity as $n_{c} \rightarrow \infty$. The behaviours of $s_{1}(h) / s_{0}(h)$ and $s_{1}(h)$ are similar.

Figure 6, a shows the normalized mode truncated spectrum and the normalized exact spectrum rescaled by $s_{0}(h)$ and $s_{1}(h)$ (i.e. the normalized spectrum of $H^{r}$ ) in the $v$ sector. No difference between the two is visible. In Table 1 values of the fifth normalized energy gap $\frac{k(3, h)+k(0, h)}{k(1, h)+k(0, h)}$ of the $v$ sector are listed: the values in the non-truncated case are listed in the first column, the values in the mode truncated case are listed in the second column and the rescaled non-truncated values are listed in the third column (which would be the same as the values in the second column if the renormalization condition could be satisfied exactly).

Figure 6.b shows the normalized mode truncated spectrum and the normalized exact spectrum rescaled by $s_{0}(h)$ and $s_{1}(h)$ in the $u$ sector. The $s_{0}(h), s_{1}(h)$ obtained in the $v$ sector were used for the rescaling, which corresponds to the assumption that $s_{0}(h)$ and $s_{1}(h)$ are the same for both sectors. The difference between the mode truncated and rescaled exact spectra is not visible in the figure.

We also see from Table 2 in which values of the fourth normalized energy gap $\frac{k(3, h)-k(0, h)}{k(1, h)-k(0, h)}$ of the $u$ sector are listed that the rescaling together with the above assumption works well.

We have not tried to calculate $s_{0}$ and $s_{1}$ for the $u$ sector because $\frac{E_{2}-E_{0}}{E_{1}-E_{0}}(h)$ is not invertible in this case. One way to circumvent this difficulty would be to use other energy levels $E_{i}, E_{j}, E_{k}$ for which $\frac{E_{i}-E_{k}}{E_{j}-E_{k}}(h)$ is invertible. 

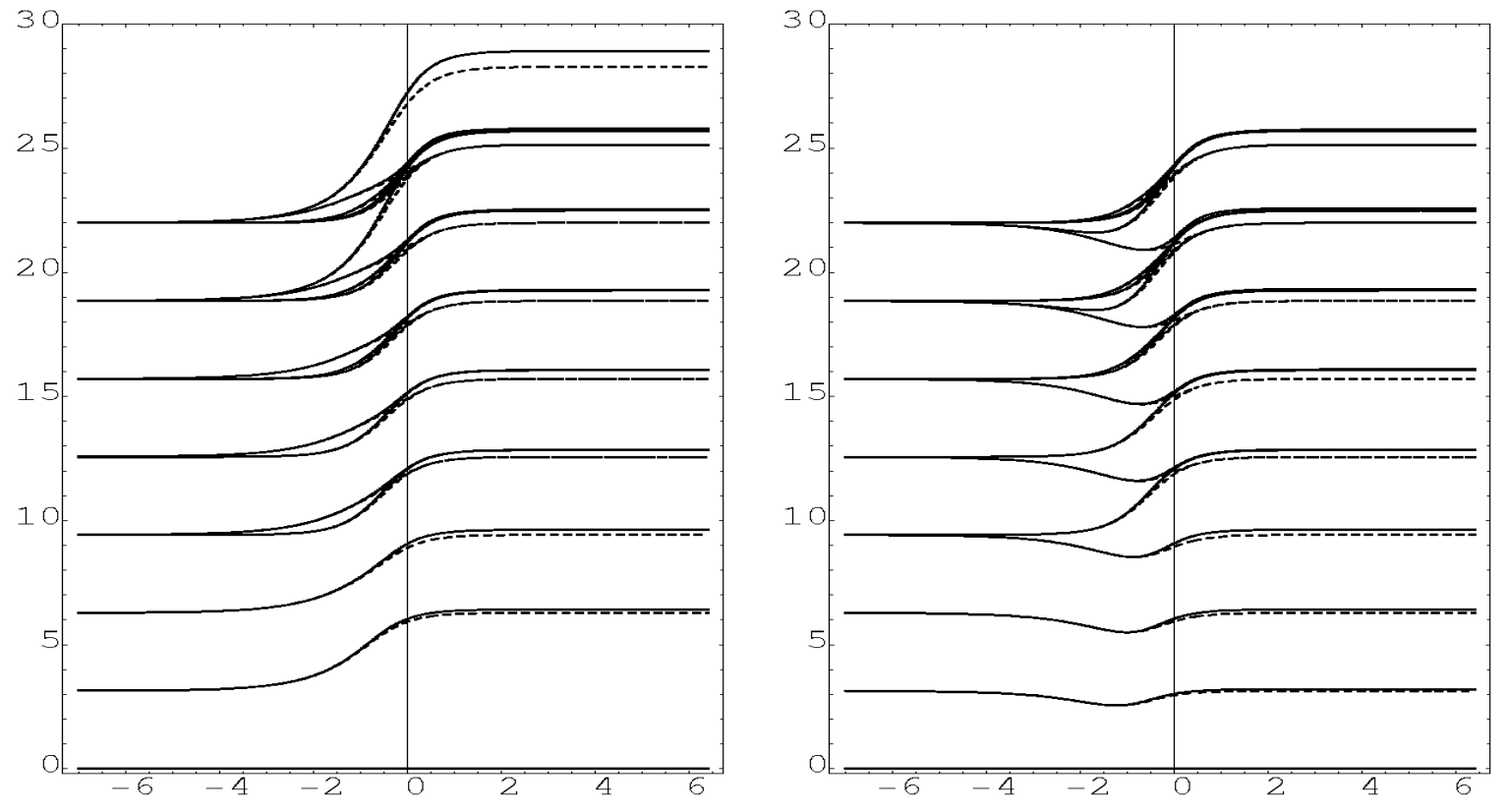

Figure 1: Exact (dashed lines) and mode truncated (solid lines) energy gaps $\left(E_{i}-E_{0}\right)$ in the $v$ and $u$ sectors respectively as a function of $\ln (h)$ at truncation level $n_{c}=9$
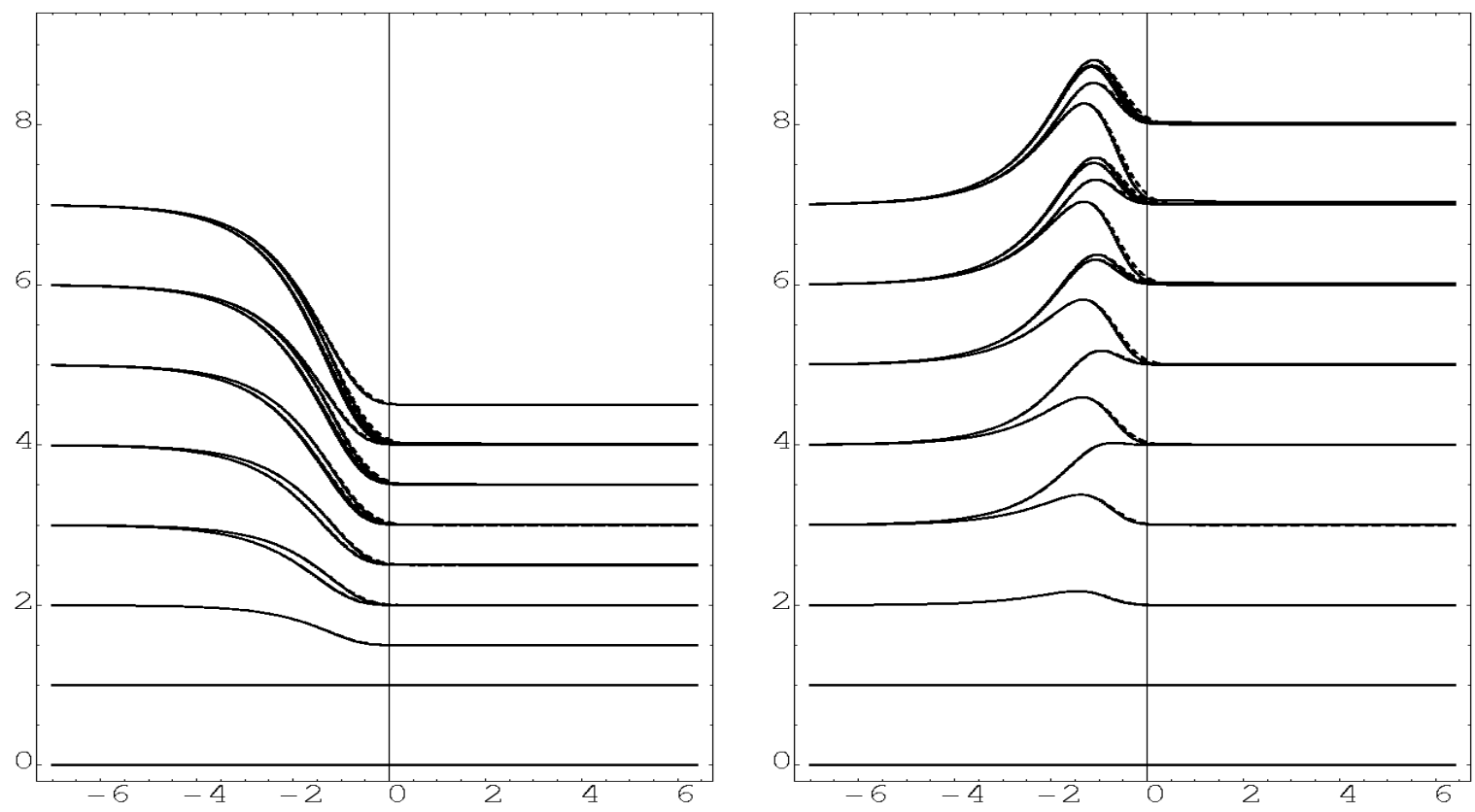

Figure 2: Exact (dashed lines) and mode truncated (solid lines) normalized spectra in the $v$ and $u$ sectors respectively as a function of $\ln (h)$ at truncation level $n_{c}=9$ 

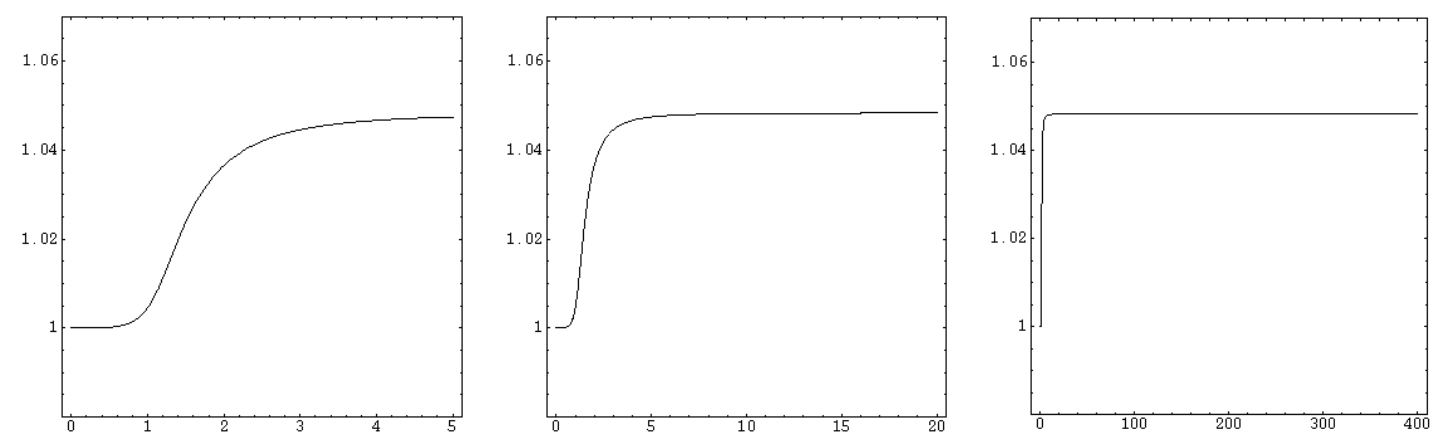

Figure 3: The function $s_{0}(h)$ for the $v$ sector in the ranges $h \in[0,3], h \in[0,20], h \in[0,400]$ and $s_{0} \in[0.95,1.05]$ at truncation level $n_{c}=9$
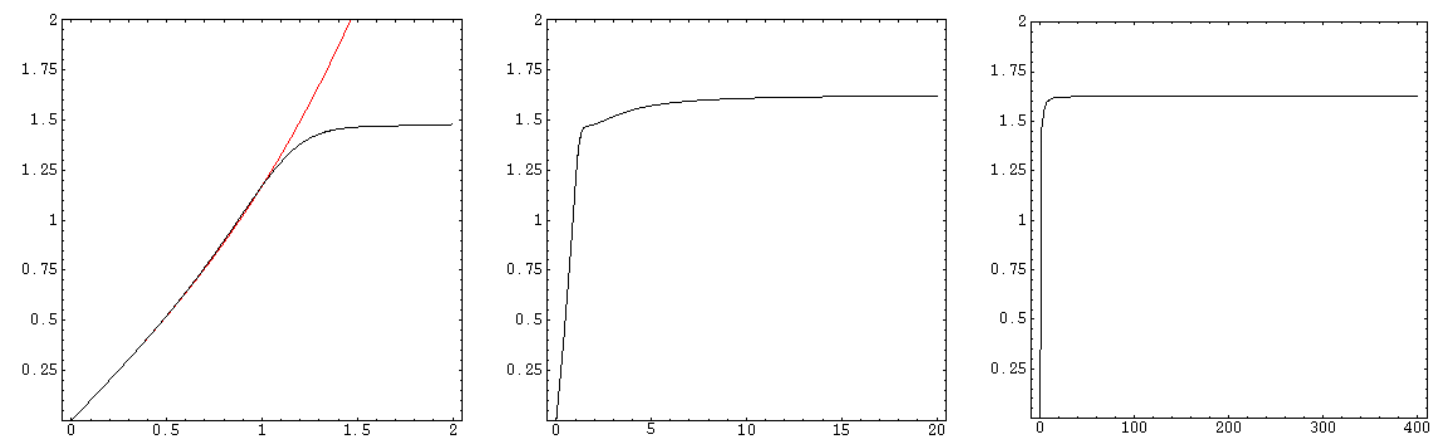

Figure 4: The function $s_{1}(h)$ for the $v$ sector in the ranges $h \in[0,2], h \in[0,20], h \in[0,400]$ and $s_{1} \in[0,2]$ at truncation level $n_{c}=9$
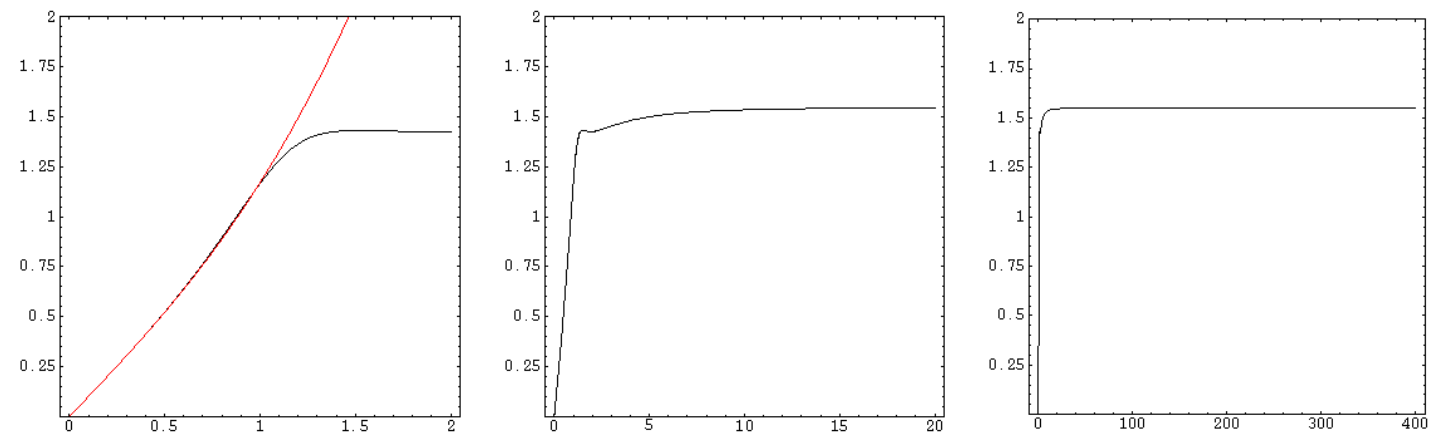

Figure 5: The function $s_{1}(h) / s_{0}(h)$ for the $v$ sector in the ranges $h \in[0,2], h \in[0,20]$, $h \in[0,400]$ and $s_{1} / s_{0} \in[0,2]$ at truncation level $n_{c}=9$ 


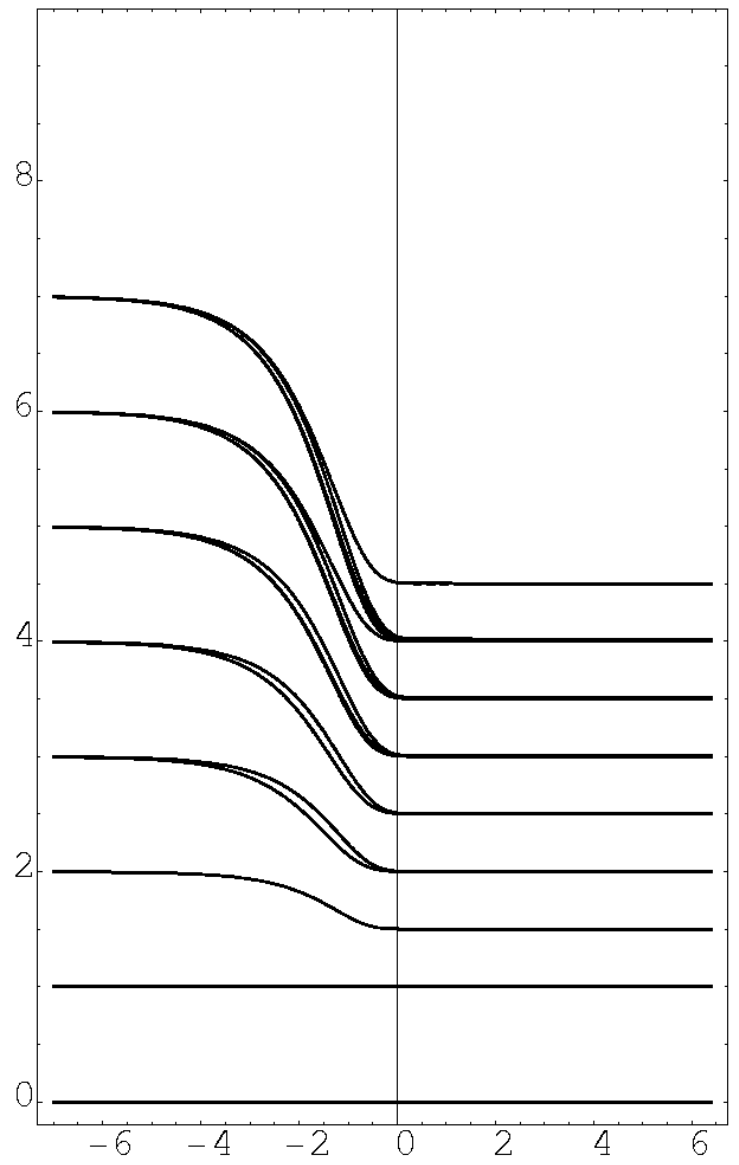

(a)

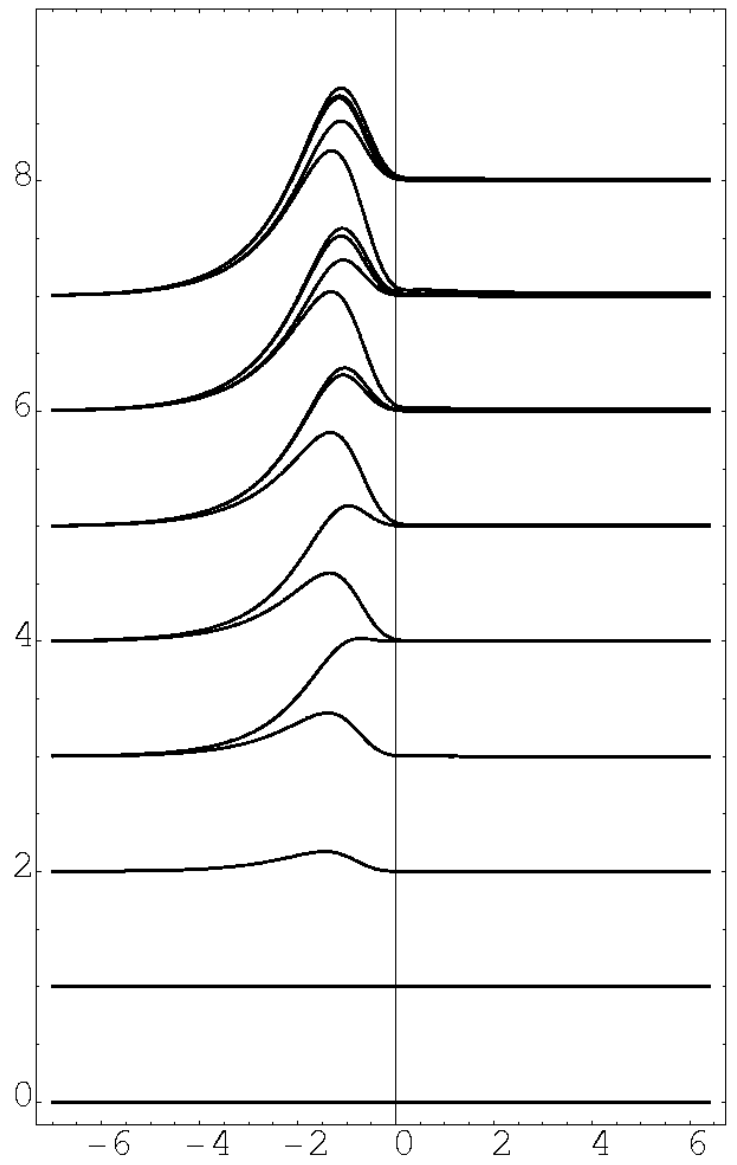

(b)

Figure 6: The mode truncated (solid lines) and rescaled exact (dashed lines) normalized spectra in the $v$ and $u$ sectors respectively as a function of $\ln (h)$ at truncation level $n_{c}=9$ 
Table 1: The normalized energy gap $\frac{k(3, h)+k(0, h)}{k(1, h)+k(0, h)}$ in the $v$ sector: exact, MT $\left(n_{c}=9\right)$ and rescaled exact values.

\begin{tabular}{clll}
\hline $\ln (h)$ & Exact & MT & Rescaled exact \\
\hline-7 & 2.997677 & 2.997677 & 2.997677 \\
-6 & 2.993681 & 2.993681 & 2.993681 \\
-5 & 2.982797 & 2.982797 & 2.982797 \\
-4 & 2.953071 & 2.953068 & 2.953068 \\
-3 & 2.8719584 & 2.8719043 & 2.8719043 \\
-2 & 2.66042177 & 2.6594328 & 2.6594328 \\
-1 & 2.25064420 & 2.24043886 & 2.24043637 \\
0 & 2.00954942 & 2.00440950 & 2.00435155 \\
1 & 2.00003474 & 2.00145821 & 2.00136637 \\
2 & 2.00000008 & 2.00106850 & 2.00100783 \\
3 & 2.0000000 & 2.0009872 & 2.0009201 \\
4 & 2.0000000 & 2.0009756 & 2.0009202 \\
5 & 2.0000000 & 2.0009740 & 2.0009187 \\
6 & 2.0000000 & 2.0009738 & 2.0009185 \\
\hline
\end{tabular}

Table 2: The normalized energy gap $\frac{k(3, h)-k(0, h)}{k(1, h)-k(0, h)}$ in the $u$ sector: exact, $\operatorname{MT}\left(n_{c}=9\right)$ and rescaled exact values.

\begin{tabular}{clll}
\hline $\ln (h)$ & Exact & MT & Rescaled exact \\
\hline-7 & 3.002321 & 3.002321 & 3.002321 \\
-6 & 3.006305 & 3.006305 & 3.006305 \\
-5 & 3.017105 & 3.017105 & 3.017105 \\
-4 & 3.046201 & 3.046203 & 3.046203 \\
-3 & 3.1225067 & 3.1225559 & 3.1225559 \\
-2 & 3.29172833 & 3.29237422 & 3.29237405 \\
-1 & 3.32029283 & 3.31272102 & 3.31271418 \\
0 & 3.01716419 & 3.00801374 & 3.00788791 \\
1 & 3.00006366 & 3.00269022 & 3.00249222 \\
2 & 3.0000000 & 3.0019707 & 3.0018400 \\
3 & 3.0000000 & 3.0018211 & 3.0017004 \\
4 & 3.0000000 & 3.0017997 & 3.0016804 \\
5 & 3.0000000 & 3.0017968 & 3.0016776 \\
6 & 3.0000000 & 3.0017964 & 3.0016773 \\
\hline
\end{tabular}




\subsection{TCS scheme}

Figure 7 shows the exact and TCSA spectra as a function of the logarithm of the coupling constant. The truncation level is $n_{c}=14$, and the dimension of the Hilbert space is 110 in each sector. It is remarkable that there is strong deviation between the TCSA and exact spectra for large values of $h$. The behaviour of the TCSA energy gaps is $E_{i}(h)-E_{0}(h) \propto h$ for large values of $h$.

Figure 8 shows the same spectra, but the lowest gap is normalized to 1, i.e. the functions $\frac{E_{i}(h)-E_{0}(h)}{E_{1}(h)-E_{0}(h)}$ are shown. It is remarkable that the agreement between the exact and TCSA spectra looks better than in the case of not normalized spectra. The functions $\frac{E_{i}(h)-E_{0}(h)}{E_{1}(h)-E_{0}(h)}$ have finite limit as $h \rightarrow \infty$ and the degeneracy pattern in this limit appears to correspond to the $c=1 / 2, h=1 / 16$ representation of the Virasoro algebra. The correspondence improves as $n_{c}$ is increased (this improvement is not illustrated). At any fixed finite value of $h$, however, the TCSA data are expected to converge to the exact values as $n_{c} \rightarrow \infty$.

Figures 9-11 show the functions $s_{0}(h), s_{1}(h), s_{1}(h) / s_{0}(h)$ in various ranges calculated in the same way as in the mode truncated case. The figures also show the curves given by (92), (93) on the left-hand side (red/grey line). It is remarkable that $s_{0}(h) \propto h$ for large values of $h$. Calculations at other values of $n_{c}$ show that the slope of $s_{0}(h)$ decreases as $n_{c}$ is increased and it can be expected to converge to 0 as $n_{c} \rightarrow \infty$. $s_{1}(h)$ appears to tend to a constant for moderately large values of $h$. Calculations at other values of $n_{c}$ show that this constant increases as $n_{c}$ is increased and it can be expected to converge to infinity as $n_{c} \rightarrow \infty$. For large values of $h, s_{1}(h)$ decreases. $s_{1}(h) / s_{0}(h)$ reaches a maximum at $h \approx 1.6$ and then decreases to zero. Calculations at other values of $n_{c}$ show that the maximum value and the value of $h$ where it is reached increase as $n_{c}$ is increased and it can be expected that both values converge to infinity as $n_{c} \rightarrow \infty$.

Figure 12. a shows the normalized TCSA spectrum and the normalized exact spectrum rescaled by $s_{0}(h)$ and $s_{1}(h)$ (i.e. the normalized spectrum of $H^{r}$ ). They show good qualitative agreement.

Values of the fifth normalized energy gap $\frac{k(3, h)+k(0, h)}{k(1, h)+k(0, h)}$ of the $v$ sector are listed in Table 3 as in the mode truncated case. These data show that the rescaling significantly improves the agreement between the TCSA and exact spectra (which is especially noticeable if $\ln (h)>-2)$.

Figure 12.b shows the normalized TCSA spectrum and the normalized exact spectrum rescaled by $s_{0}(h)$ and $s_{1}(h)$ in the $u$ sector. The $s_{0}(h), s_{1}(h)$ functions obtained in the $v$ sector were used for the rescaling as in the mode truncated case. Table 4 shows values of the fourth normalized energy gap $\frac{k(3, h)-k(0, h)}{k(1, h)-k(0, h)}$ of the $u$ sector. We see from the table that the assumption that $s_{0}(h)$ and $s_{1}(h)$ are the same in both sectors does not give very good result in this case, although the situation might become better at higher truncation levels. 

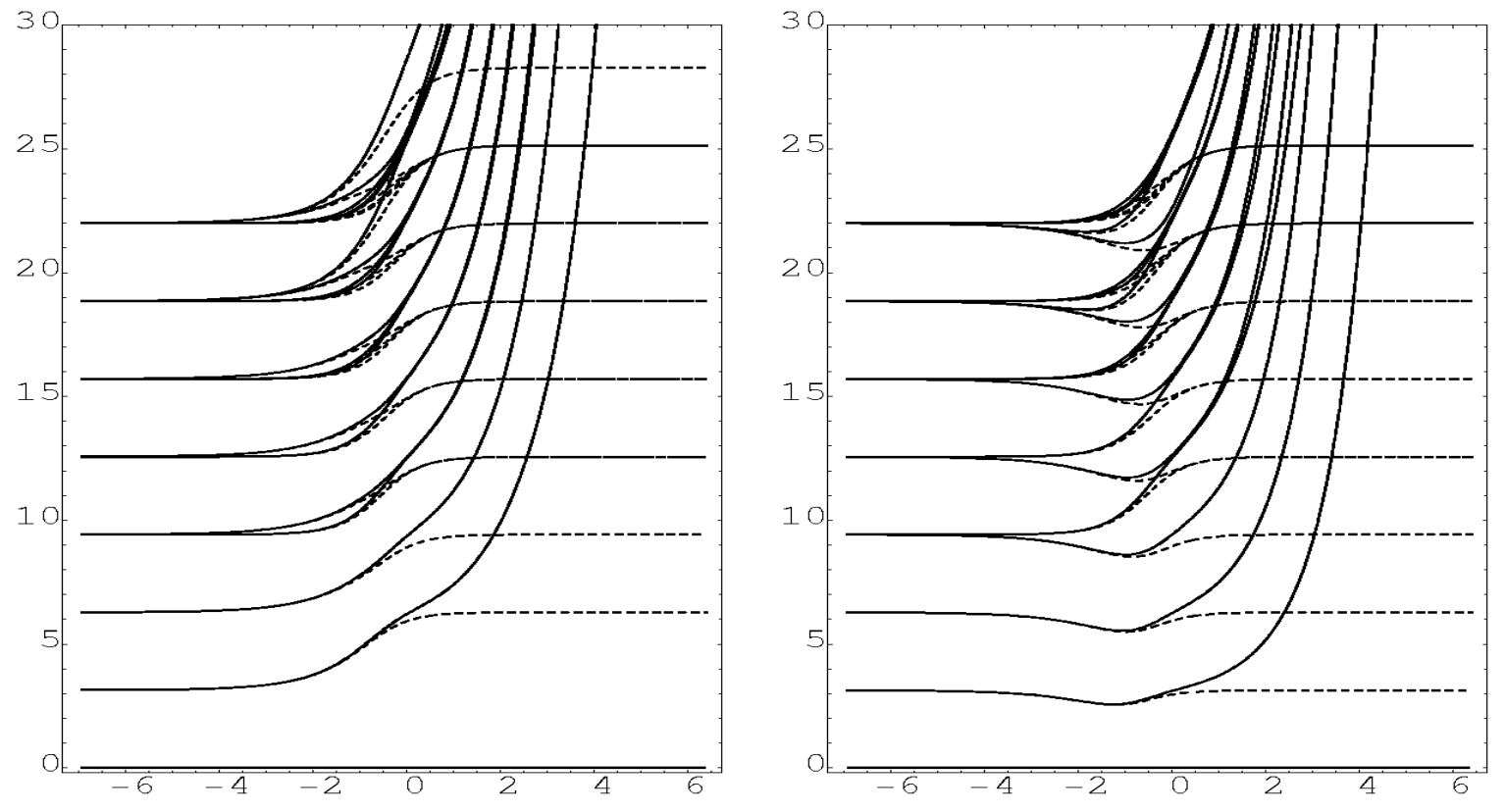

Figure 7: Exact (dashed lines) and TCSA (solid lines) energy gaps $\left(E_{i}-E_{0}\right)$ in the $v$ and $u$ sectors respectively as a function of $\ln (h)$ at truncation level $n_{c}=14$
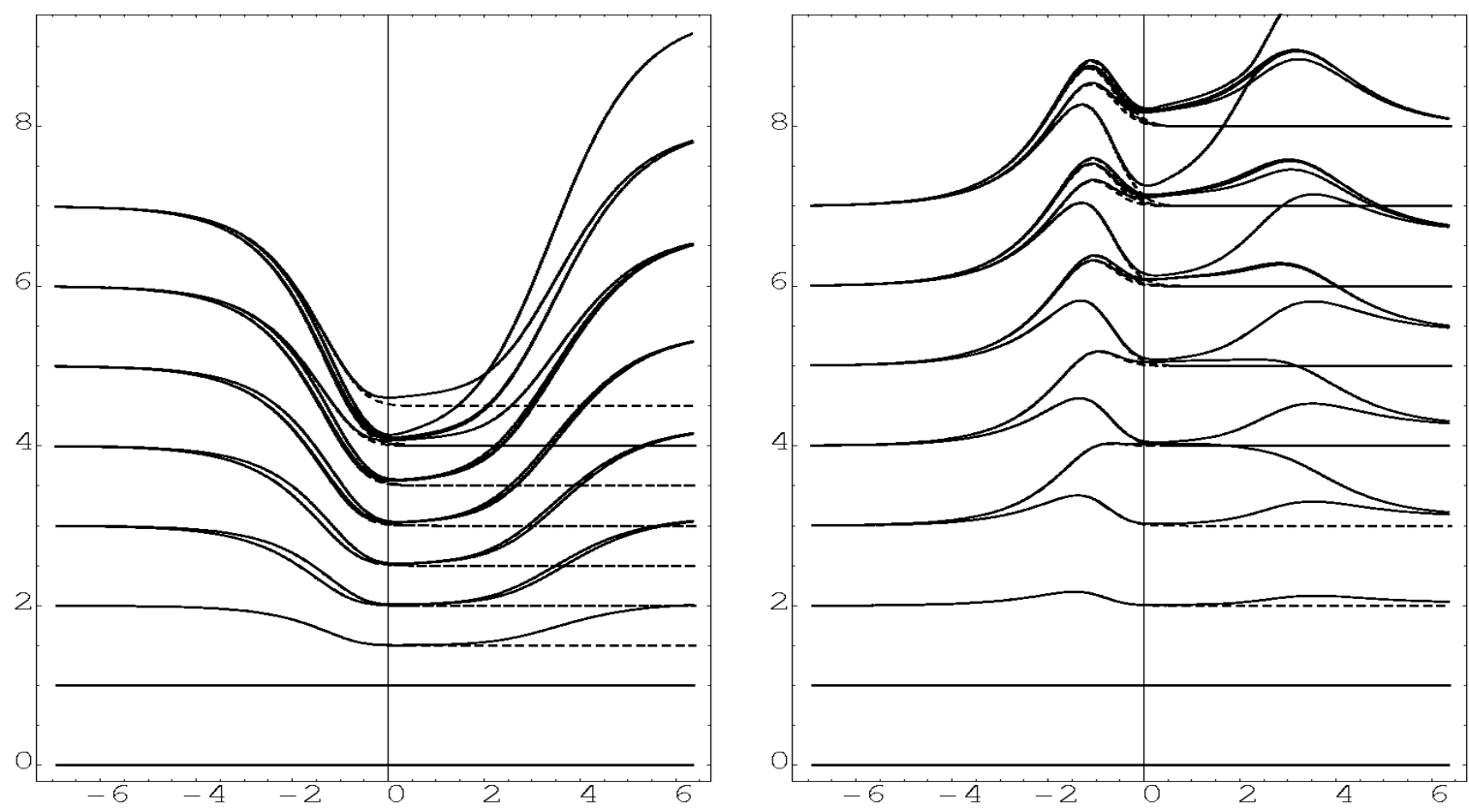

Figure 8: Exact (dashed lines) and TCSA (solid lines) normalized spectra in the $v$ and $u$ sectors respectively as a function of $\ln (h)$ at truncation level $n_{c}=14$ 

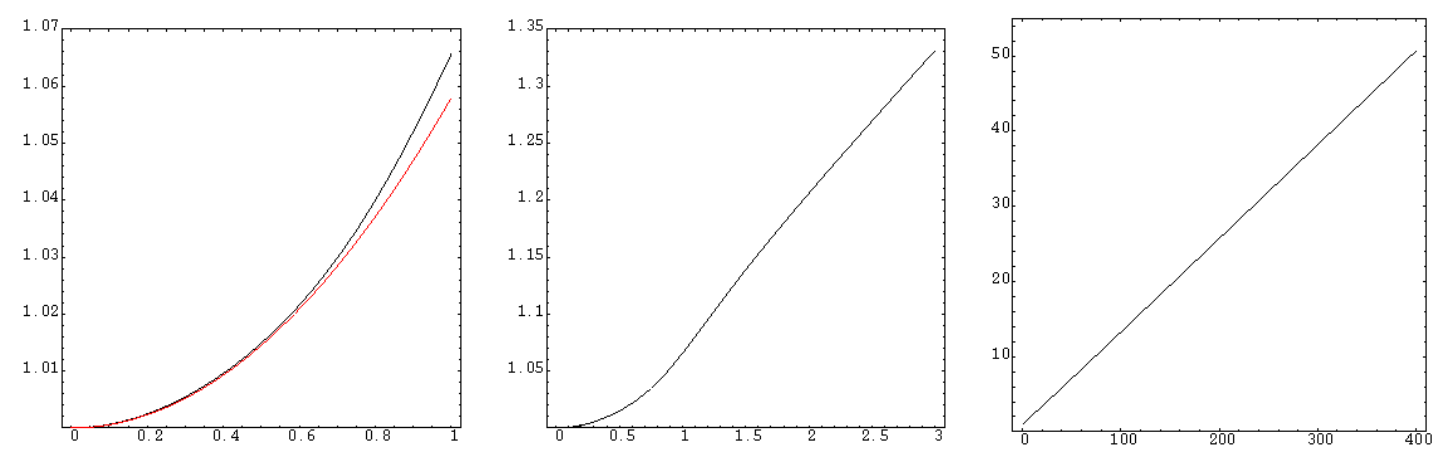

Figure 9: The function $s_{0}(h)$ for the $v$ sector in the ranges $h \in[0,1], s_{0} \in[1,1.07] ; h \in[0,3]$, $s_{0} \in[1,1.35] ; h \in[0,400], s_{0} \in[1,60]$ at truncation level $n_{c}=14$
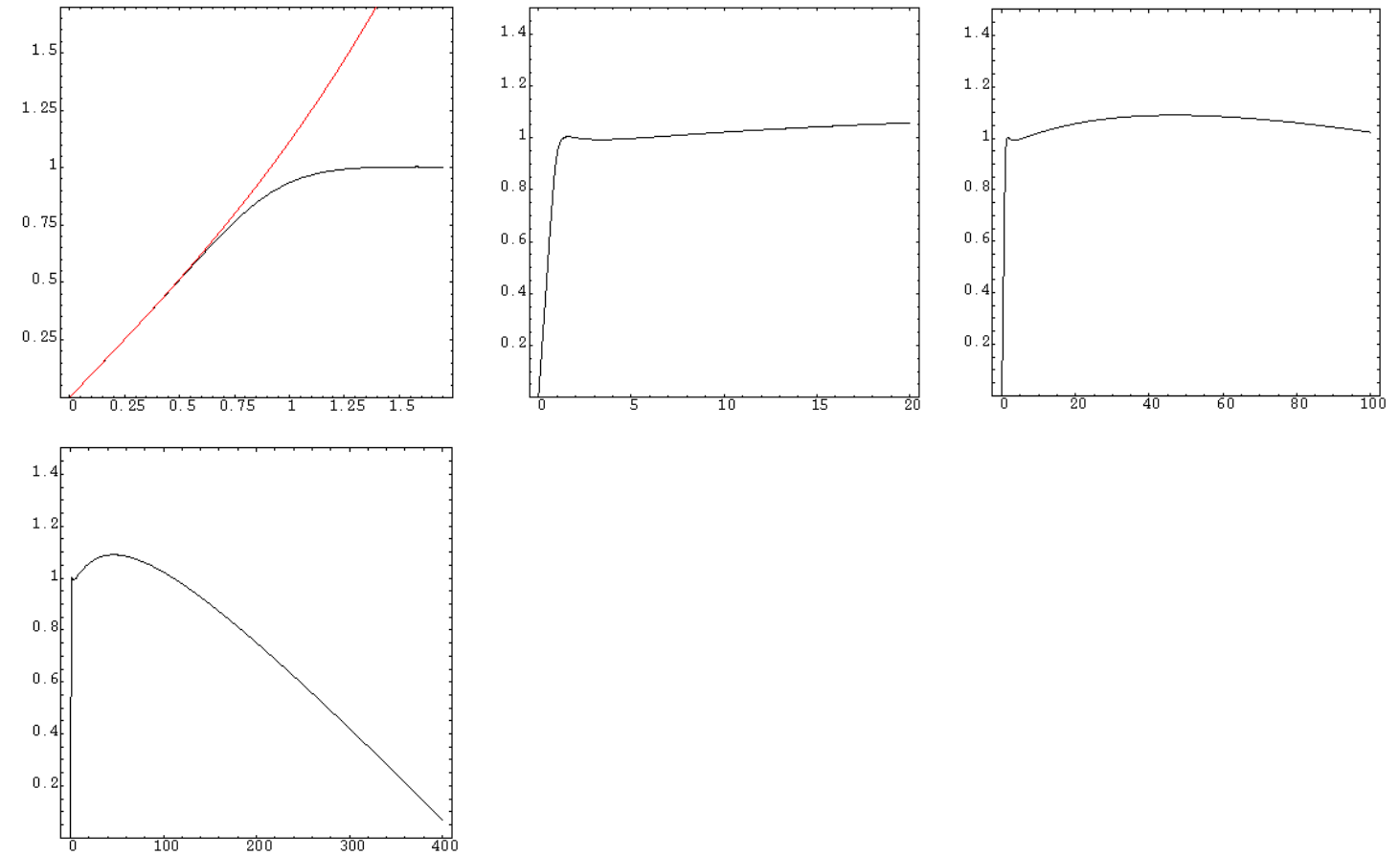

Figure 10: The function $s_{1}(h)$ for the $v$ sector in the ranges $h \in[0,1.75], s_{1} \in[0,1.7]$; $h \in[0,20], s_{1} \in[0,1.5] ; h \in[0,100], s_{1} \in[0,1.5] ; h \in[0,400], s_{1} \in[0,1.5]$ at truncation level $n_{c}=14$ 

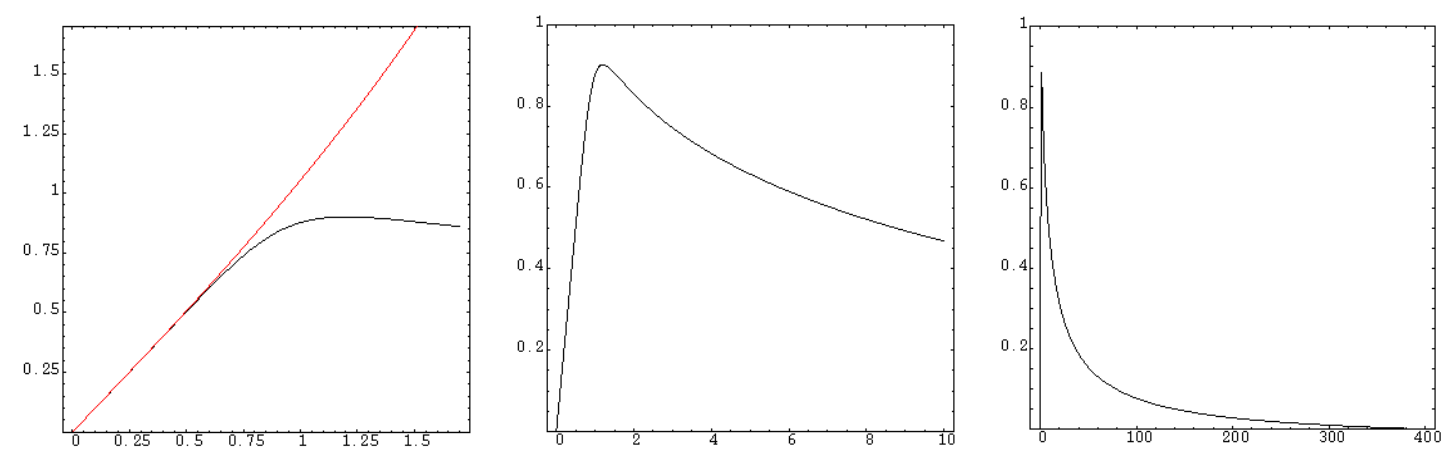

Figure 11: The function $s_{1}(h) / s_{0}(h)$ for the $v$ sector in the ranges $h \in[0,1.75], s_{1} / s_{0} \in$ $[0,1.75] ; h \in[0,10], s_{1} / s_{0} \in[0,1] ; h \in[0,400], s_{1} / s_{0} \in[0,1]$ at truncation level $n_{c}=14$

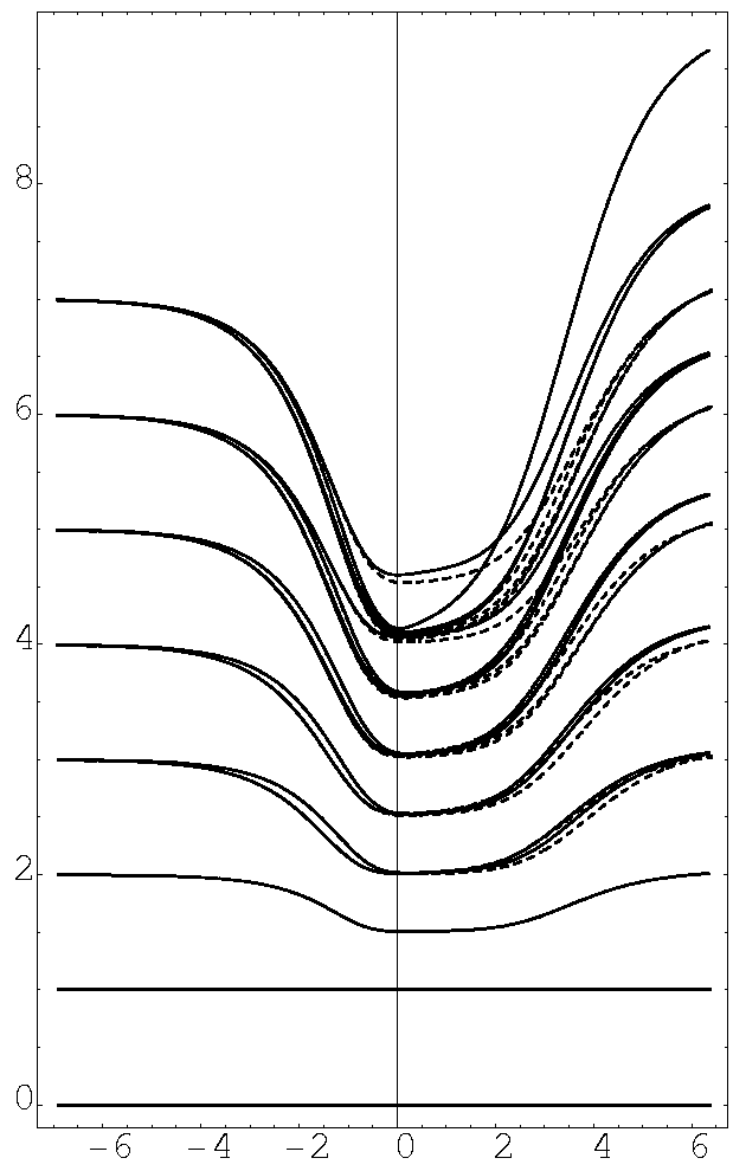

(a)

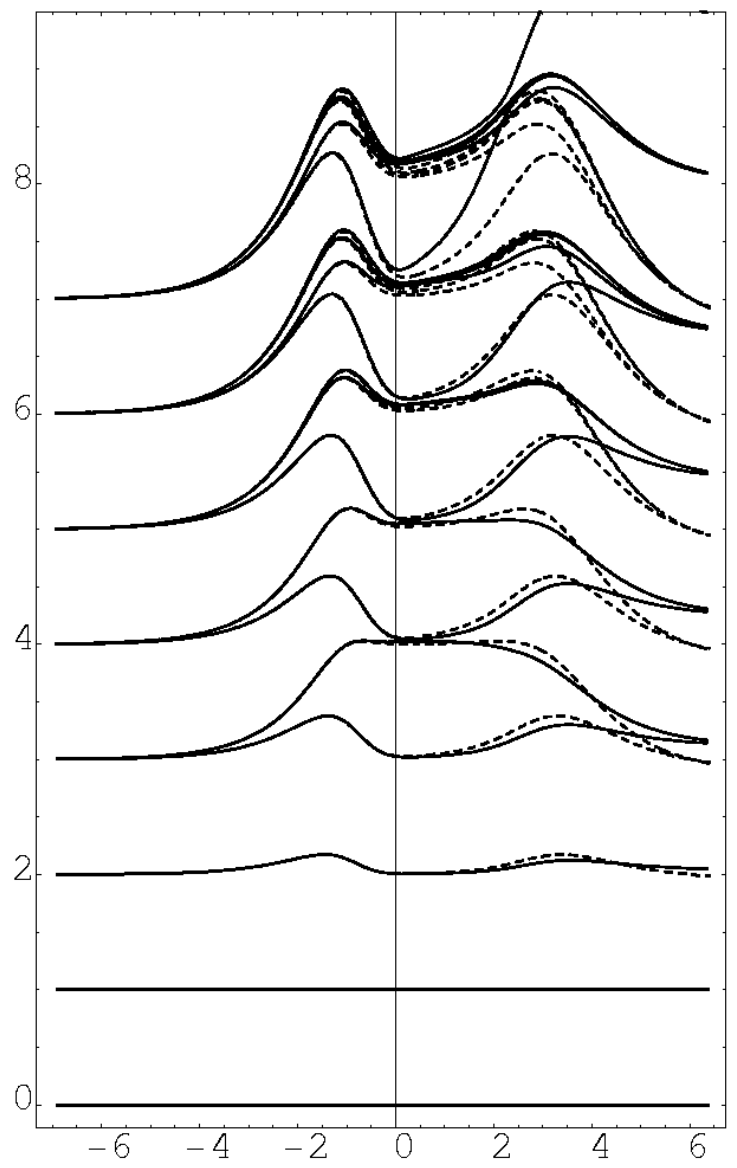

(b)

Figure 12: The TCSA (solid lines) and rescaled exact (dashed lines) normalized spectra in the $v$ and $u$ sectors respectively as a function of $\ln (h)$ at truncation level $n_{c}=14$ 
Table 3: The normalized energy gap $\frac{k(3, h)+k(0, h)}{k(1, h)+k(0, h)}$ in the $v$ sector: exact, TCSA $\left(n_{c}=14\right)$ and rescaled exact values.

\begin{tabular}{clll}
\hline $\ln (h)$ & Exact & TCSA & Rescaled exact \\
\hline-6 & 2.993681 & 2.993681 & 2.993681 \\
-5 & 2.982797 & 2.982797 & 2.982797 \\
-4 & 2.953071 & 2.953074 & 2.953073 \\
-3 & 2.8719584 & 2.871974 & 2.871961 \\
-2 & 2.66042177 & 2.660322 & 2.660236 \\
-1 & 2.25064420 & 2.2488 & 2.24837 \\
0 & 2.00954942 & 2.01699 & 2.0177 \\
1 & 2.00003474 & 2.028321 & 2.031631 \\
2 & 2.00000008 & 2.101337 & 2.105516 \\
3 & 2.0000000 & 2.33433 & 2.32573 \\
4 & 2.0000000 & 2.670454 & 2.643480 \\
5 & 2.0000000 & 2.916318 & 2.879339 \\
6 & 2.0000000 & 3.037542 & 2.997132 \\
\hline
\end{tabular}

Table 4: The normalized energy gap $\frac{k(3, h)-k(0, h)}{k(1, h)-k(0, h)}$ in the $u$ sector: exact, TCSA $\left(n_{c}=14\right)$ and rescaled exact values.

\begin{tabular}{clll}
\hline $\ln (h)$ & Exact & TCSA & Rescaled exact \\
\hline-7 & 3.002321 & 3.002321 & 3.002321 \\
-6 & 3.006305 & 3.006305 & 3.006305 \\
-5 & 3.017105 & 3.017105 & 3.017105 \\
-4 & 3.046201 & 3.046206 & 3.046198 \\
-3 & 3.1225067 & 3.122558 & 3.122504 \\
-2 & 3.29172833 & 3.292196 & 3.291849 \\
-1 & 3.32029283 & 3.31947 & 3.31865 \\
0 & 3.01716419 & 3.0268 & 3.0315 \\
1 & 3.00006366 & 3.03610 & 3.05522 \\
2 & 3.0000000 & 3.11033 & 3.16799 \\
3 & 3.0000000 & 3.27063 & 3.36158 \\
4 & 3.0000000 & 3.28320 & 3.30254 \\
5 & 3.0000000 & 3.203134 & 3.115763 \\
6 & 3.0000000 & 3.152931 & 3.002865 \\
\hline
\end{tabular}




\section{Discussion}

We have investigated the validity of the approach (5) for the description of truncation effects in TCSA spectra. Comparison with another truncation method called mode truncation shows that the remarkably regular behaviour of the TCSA spectrum for large $h$ in the case of the model (2), namely the presence of second flows, is not universal (i.e. not independent of the truncation scheme). The numerical calculations show that (5) provides a good approximation of the truncated spectra in both the TCSA and the mode truncation scheme. This is confirmed by perturbative analytic calculations as well. The main difference between the mode truncated and TCSA spectra at large $h$ seems to be explicable through the different behaviour of the function $s_{0}\left(n_{c}, h\right)$ in the two schemes. Difference between the $s_{0}\left(n_{c}, h\right)$ functions appears also in perturbation theory. We have shown analytically that in the mode truncation scheme the convergence of the truncated spectra to the exact spectra can be improved by (at least) one order in $1 / n_{c}$ by the rescaling (5). This has also been shown in the TCSA scheme for low orders of perturbation theory in $h$.

We have also given a quantum field theoretic discussion of the model (2). In particular we have discussed (see Appendix $\mathrm{B}$ ) the change of the boundary condition satisfied by the fermion fields as the coupling constant (or external boundary magnetic field) is increased. Such a change, which is emphasized in the literature, seems impossible naively — in our formulation at least. The paradox is resolved by the phenomenon that the fermion fields (more precisely their matrix elements between energy eigenstates) develop a discontinuity at the boundary if the coupling constant is nonzero.

It is still an open problem to present an explanation of the validity of the approach (5). Within the framework of (5) the behaviour of the TCSA spectrum at large $h$, in particular the second flow mentioned in the Introduction, is explained by the behaviour of $s_{0}$ and $s_{1}$ at large $h: s_{0} \propto h, s_{1}$ is bounded from above, therefore $s_{1} / s_{0}$ tends to zero. It is a further problem to give an analytic derivation of this behaviour of $s_{0}$ and $s_{1}$.

We shall investigate the scaling properties of $s_{1} / s_{0}, s_{1}$ and $s_{0}$ and the TCSA and MT spectra in a further publication [33]. It is also interesting to extend the investigations to other perturbed boundary conformal minimal models, which show similar behaviour numerically to the model that we have studied. Certain results concerning other minimal models and scaling properties already exist [25, 20]; see also [18]. It is a further problem to classify the possible behaviours of truncated spectra at large $h$ for various truncation schemes. Finally, the quantum field theoretic description of the model (2) could be developed further and extended to case of massive particles.

\section{Acknowledgments}

I would like to thank Gérard Watts for proposing the problem and for several useful discussions, Zoltán Bajnok, Gábor Takács and László Palla for useful discussions, and the 
Department of Mathematics at King's College London, where part of this work was carried out, for hospitality. I was supported by the Hungarian fund OTKA (grants T037674 and K60040), by the EUCLID research training network (HPRN-CT-2002-00325) at various times and by the Marie Curie Training Site 'Strings, Branes and Boundary Conformal Field Theory' (MCFH-2001-00296) at King's College London.

\section{A Distributions on closed line segments}

The necessary formulae for the Dirac delta $\delta(x)$ and step function $\Theta(x)$ distributions on the closed interval $[0, L] \subset \mathbb{R}$ are the following:

$$
\begin{array}{ll}
\int_{0}^{L} \delta(x-a) f(x) \mathrm{d} x=f(a) & \text { if } \quad a \in(0, L) \\
\int_{0}^{L} \delta(x-a) f(x) \mathrm{d} x=\frac{1}{2} f(0) & \text { if } \quad a=0 \\
\int_{0}^{L} \delta(x-a) f(x) \mathrm{d} x=\frac{1}{2} f(L) & \text { if } \quad a=L \\
\int_{0}^{L} \delta(x-a) f(x) \mathrm{d} x=0 & \text { if } \quad a \notin[0, L],
\end{array}
$$

where $f$ is a function defined on $[0, L]$, and $x \in[0, L]$.

$$
\Theta(x-a)=0 \quad \text { if } \quad x<a, \quad \Theta(x-a)=1 \quad \text { if } \quad x \geq a,
$$

where $a \in \mathbb{R}$,

$$
\begin{aligned}
& \partial_{x} \Theta(x-L)=2 \delta(x-L) \\
& \partial_{x} \Theta(x-a)=\delta(x-a) \quad \text { if } \quad a \in(0, L) \\
& \partial_{x} \Theta(x-a)=0 \quad \text { if } a \notin(0, L] \text {, }
\end{aligned}
$$

where $x \in[0, L]$.

$$
\begin{gathered}
\sum_{k \in \frac{\pi \mathbb{Z}}{L}} \exp \left[\mathrm{i} k\left(x-x^{\prime}\right)\right]=2 L \delta\left(x-x^{\prime}\right) \\
\sum_{k \in \frac{\pi \mathbb{Z}}{L}} \exp \left[\mathrm{i} k\left(x+x^{\prime}\right)\right]=2 L\left[\delta\left(x+x^{\prime}\right)+\delta\left(x+x^{\prime}-2 L\right)\right]
\end{gathered}
$$

where $x, x^{\prime} \in[0, L]$. 


\section{B Extension to Section 2}

In this appendix we present further details for Section 2 ,

The anticommutators of the $b(k)$ can be obtained in the following way: we define a scalar product on the classical complex valued solutions of the equations of motion:

$$
\left\langle\psi_{1}, \psi_{2}, a_{2} \mid \phi_{1}, \phi_{2}, b_{2}\right\rangle=\int_{0}^{L}\left[\psi_{1}^{*} \phi_{1}+\psi_{2}^{*} \phi_{2}\right] \mathrm{d} x+2 L a_{2}^{*} b_{2}
$$

This product should be calculated at a fixed time. Using the equations of motion it can be shown that the product is independent of this time.

The essential properties of this scalar product are that it is defined by a local expression and that the $n(k)$ are orthogonal with respect to it:

$$
\left\langle n\left(k_{1}\right) \mid n\left(k_{2}\right)\right\rangle=\delta_{k_{1}-k_{2}, 0}\left(2 L+\frac{\sin \left(2 k_{1} L\right)}{k_{1}}\right) .
$$

The creation/annihilation operators can be expressed in the following way:

$$
\left\langle n(k) \mid\left(\Psi_{1}, \Psi_{2}, A_{2}\right)\right\rangle=b(k)\langle n(k) \mid n(k)\rangle
$$

Using the formula (108) and the anticommutation relations (6)

$$
\left\{b\left(k_{1}\right), b\left(k_{2}\right)\right\}=\delta_{k_{1}+k_{2}, 0} \frac{4 L k_{1}}{2 L k_{1}+\sin \left(2 L k_{1}\right)} .
$$

We remark that the above scalar product technique is also suitable for free fields on the half-line or in the usual full Minkowski space without boundaries in arbitrary spacetime dimensions.

The nonzero matrix elements of the fields are

$$
\begin{aligned}
& \frac{\left\langle P\left|\left(\Psi_{1}(x, t), \Psi_{2}(x, t), A_{2}(t)\right)\right| Q\right\rangle}{\sqrt{|\langle P \mid P\rangle\langle Q \mid Q\rangle|}}=n(k)(-1)^{m} \sqrt{f(k)} \\
& \frac{\left\langle Q\left|\left(\Psi_{1}(x, t), \Psi_{2}(x, t), A_{2}(t)\right)\right| P\right\rangle}{\sqrt{|\langle P \mid P\rangle\langle Q \mid Q\rangle|}}=n(-k)(-1)^{m} \sqrt{f(k)},
\end{aligned}
$$

where $|Q\rangle=b\left(k_{1}\right) b\left(k_{2}\right) \ldots b\left(k_{n}\right)\left|0_{h}\right\rangle,|P\rangle=b\left(k_{1}\right) \ldots b\left(k_{m}\right) b(k) b\left(k_{m+1}\right) \ldots b\left(k_{n}\right)\left|0_{h}\right\rangle$,

$$
\begin{gathered}
f(k)=\frac{4 L k}{2 L k+\sin (2 L k)}, \\
\langle Q \mid Q\rangle=\prod_{i=1}^{n} f\left(k_{i}\right) .
\end{gathered}
$$


The Hamiltonian operator can be written as

$$
H=\sum_{k \in S, k>0} \frac{k}{f(k)} b(k) b(-k) .
$$

The following formula can be written for $\left|0_{h}\right\rangle$ :

$$
N\left|0_{h}\right\rangle=\lim _{\alpha \rightarrow \infty} \mathrm{e}^{-\alpha H}|v\rangle=\prod_{k \in S, k>0}\left(1-\frac{1}{f(k)} b(k) b(-k)\right)|v\rangle,
$$

where $N$ is a normalization factor. The second equation on the right-hand side can be verified directly using the following formulae: $\left[b\left(k_{1}\right) b\left(-k_{1}\right), b\left(k_{2}\right) b\left(-k_{2}\right)\right]=0$ if $k_{1} \neq k_{2}$ (and $\left.k_{1}, k_{2}>0\right)$, and $(b(k) b(-k))^{n}=f(k)^{n-1} b(k) b(-k)$.

We remark that we have not given a mathematically completely rigorous proof that (43) satisfies (6) -(9), but we think that this would be possible.

The expansion (43) and (6)-(9) imply that the following formulae hold:

$$
\begin{aligned}
\sum_{k \in S} f(k) \psi_{1}(k)(x, 0) \psi_{1}(-k)(y, 0) & =4 L \delta(x-y) \\
\sum_{k \in S} f(k) \psi_{2}(k)(x, 0) \psi_{2}(-k)(y, 0) & =4 L \delta(x-y) \\
\sum_{k \in S} f(k) \psi_{1}(k)(x, 0) \psi_{2}(-k)(y, 0) & =-4 L[\delta(x+y)+\delta(x+y-2 L)] \\
\sum_{k \in S} f(k) a_{2}(k)(0) \psi_{1}(-k)(x, 0) & =0 \\
\sum_{k \in S} f(k) a_{2}(k)(0) \psi_{2}(-k)(x, 0) & =0 \\
\sum_{k \in S} f(k) a_{2}(k)(0) a_{2}(-k)(0) & =2 .
\end{aligned}
$$

These formulae are generalizations of (106), (107).

Using the formulae

$$
\begin{aligned}
a(k) & =\frac{1}{2 \sqrt{2} L}\left[\int_{0}^{L} \mathrm{e}^{\mathrm{i} k x} \Psi_{1}(x, 0) \mathrm{d} x-\int_{0}^{L} \mathrm{e}^{-\mathrm{i} k x} \Psi_{2}(x, 0) \mathrm{d} x\right] \\
A_{1} & =\frac{1}{2 L}\left[\int_{0}^{L} \Psi_{1}(x, 0) \mathrm{d} x-\int_{0}^{L} \Psi_{2}(x, 0) \mathrm{d} x\right]
\end{aligned}
$$


and (43) we obtain the following relations:

$$
\begin{aligned}
a(k) & =\frac{-1}{\sqrt{2} L} \sum_{k^{\prime} \in S} b\left(k^{\prime}\right) \frac{\sin \left[\left(k-k^{\prime}\right) L\right]}{k-k^{\prime}} \\
A_{1} & =\frac{-1}{L} \sum_{k^{\prime} \in S} b\left(k^{\prime}\right) \frac{\sin \left[k^{\prime} L\right]}{k^{\prime}} \\
A_{2}(0) & =\sum_{k \in S} b(k) \frac{-\mathrm{i} \sin (k L)}{4 L h}=-\mathrm{i} \sum_{k \in S} b(k) \frac{\sin (k L)}{\sqrt{k L \tan (k L)}} .
\end{aligned}
$$

Using (110) we get the relation

$$
\begin{array}{r}
b(k)\left(2 L+\frac{\sin (2 k L)}{k}\right)=\sqrt{2} \sum_{k^{\prime} \in \frac{\pi}{L} \mathbb{Z}} a\left(k^{\prime}\right) \\
+2 \sin \left[\left(k^{\prime}-k\right) L\right] \\
k^{\prime}-k \\
+\frac{i \sin (k L)}{2 h} A_{2}(0) .
\end{array}
$$

(126)-(129) can be regarded as Bogoliubov transformation formulae for this model.

In the $h \rightarrow 0$ limit

$$
\begin{array}{rlr}
b(k(n, h)) & \rightarrow-\sqrt{2} a(k(n, 0)) & \\
b(k(0, h))+b(k(0, h))^{\dagger} & \rightarrow-A_{1} \\
\mathrm{i}\left(b(k(0, h))^{\dagger}-b(k(0, h))\right) & \rightarrow A_{2}(0) & \\
\left|0_{h}\right\rangle & \rightarrow|v\rangle . &
\end{array}
$$

The following boundary conditions are satisfied:

$$
\left\langle E_{1}\left|\Psi_{1}(0, t)+\Psi_{2}(0, t)\right| E_{2}\right\rangle=0 \quad\left\langle E_{1}\left|\Psi_{1}(L, t)+\Psi_{2}(L, t)\right| E_{2}\right\rangle=0
$$

and

$$
\begin{aligned}
& \lim _{x \rightarrow 0}\left\langle E_{1}\left|\Psi_{1}(x, t)+\Psi_{2}(x, t)\right| E_{2}\right\rangle \quad=0 \\
& \lim _{x \rightarrow L}\left\langle E_{1}\left|\partial_{x} \Psi_{1}(x, t)-\partial_{x} \Psi_{2}(x, t)\right| E_{2}\right\rangle \\
& =16 L h^{2}\left\langle E_{1}\left|\Psi_{2}(L, t)-\Psi_{1}(L, t)\right| E_{2}\right\rangle \\
& \lim _{h \rightarrow \infty} \lim _{x \rightarrow L}\left\langle E_{1}\left|\Psi_{1}(x, t)-\Psi_{2}(x, t)\right| E_{2}\right\rangle=0 \\
& \lim _{h \rightarrow \infty} \lim _{x \rightarrow L}\left\langle E_{1}\left|\Psi_{1}(x, t)+\Psi_{2}(x, t)\right| E_{2}\right\rangle \neq 0,
\end{aligned}
$$

where $\left|E_{1}\right\rangle$ and $\left|E_{2}\right\rangle$ are eigenstates of $H$. The boundary conditions (134) are the same as those satisfied by the free fields. On the other hand, (135) and (136) are similar to the 
boundary conditions written down in $[23,27,26]$. From the point of view of the boundary conditions one can say that the perturbation $H_{I}$ induces a flow from the boundary condition $\lim _{x \rightarrow L}\left\langle E_{1}\left|\Psi_{1}(x, t)+\Psi_{2}(x, t)\right| E_{2}\right\rangle=0$ to the boundary condition $\lim _{x \rightarrow L}\left\langle E_{1}\right| \Psi_{1}(x, t)-$ $\Psi_{2}(x, t)\left|E_{2}\right\rangle=0$ and the boundary condition on the left-hand side remains constant, which is in accordance with the literature (see e.g. [23]). The boundary condition $\left\langle E_{1}\right| \Psi_{1}(L, t)+$ $\Psi_{2}(L, t)\left|E_{2}\right\rangle=0$ is called free spin boundary condition in the literature (e.g. [23]) and $\left\langle E_{1}\left|\Psi_{1}(L, t)-\Psi_{2}(L, t)\right| E_{2}\right\rangle=0$ is called fixed spin boundary condition.

We remark that from (135) and (136) and the bulk equations of motion $\left(\partial_{t}+\partial_{x}\right) \Psi_{1}=0$, $\left(\partial_{t}-\partial_{x}\right) \Psi_{2}=0$ equation (38) can be recovered.

We define the fields

$$
\Phi_{1}(x, t)=\sum_{k \in S}-b(k) \mathrm{e}^{\mathrm{i} k(t-x)} \quad \Phi_{2}(x, t)=\sum_{k \in S} b(k) \mathrm{e}^{\mathrm{i} k(t+x)} .
$$

They satisfy the following equations:

$$
\begin{aligned}
\left(\Psi_{1}-\Psi_{2}\right)(L, t) & =\frac{1}{16 L h^{2}} \partial_{x}\left(\Phi_{2}-\Phi_{1}\right)(L, t) \\
A_{2}(t) & =-\frac{1}{8 L h}\left(\Phi_{1}+\Phi_{2}\right)(L, t) \\
H_{0}=-\frac{\mathrm{i}}{8 L} \int_{0}^{L} \mathrm{~d} x \Phi_{1}(x, 0) \partial_{x} \Phi_{1}(x, 0) & +\frac{\mathrm{i}}{8 L} \int_{0}^{L} \mathrm{~d} x \Phi_{2}(x, 0) \partial_{x} \Phi_{2}(x, 0)-\frac{1}{2} h H_{I} .
\end{aligned}
$$

Note that the energies of the modes are not in $\pi \mathbb{Z} / L$, so one cannot conclude that the sum of the first two terms in (142) equals to $H$.

The above equations suggest how to describe the model discussed in Section 2 and in this appendix as a perturbation of the $h \rightarrow \infty$ limiting model. This description is discussed Section 3. The boundary conditions in this case are

$$
\left\langle E_{1}\left|\Phi_{1}(0, t)+\Phi_{2}(0, t)\right| E_{2}\right\rangle=0 \quad\left\langle E_{1}\left|\Phi_{1}(L, t)-\Phi_{2}(L, t)\right| E_{2}\right\rangle=0
$$

and

$$
\begin{aligned}
& \lim _{x \rightarrow 0}\left\langle E_{1}\left|\Phi_{1}(x, t)+\Phi_{2}(x, t)\right| E_{2}\right\rangle \quad=0 \\
& \lim _{x \rightarrow L}\left\langle E_{1}\left|\partial_{x} \Phi_{1}(x, t)-\partial_{x} \Phi_{2}(x, t)\right| E_{2}\right\rangle \\
& =\frac{1}{16 L g}\left\langle E_{1}\left|\Phi_{1}(L, t)-\Phi_{2}(L, t)\right| E_{2}\right\rangle \\
& \lim _{g \rightarrow \infty} \lim _{x \rightarrow L}\left\langle E_{1}\left|\Phi_{1}(x, t)+\Phi_{2}(x, t)\right| E_{2}\right\rangle=0 \\
& \lim _{g \rightarrow \infty} \lim _{x \rightarrow L}\left\langle E_{1}\left|\Phi_{1}(x, t)-\Phi_{2}(x, t)\right| E_{2}\right\rangle \neq 0,
\end{aligned}
$$

where $\left|E_{1}\right\rangle$ and $\left|E_{2}\right\rangle$ are eigenstates of $H$. 


\section{Bethe-Yang equations}

The Bethe-Yang equations can be used to give a description of the spectrum of models in finite volume which have factorized scattering in their infinite volume limit. The BetheYang equations for relativistic models defined on a cylinder are exposed, for example, in [34, 35, 36]; for models defined on the strip they are written down in [37, 38]. It should be noted that the Bethe-Yang equations usually give approximate result only.

In the case of the model that we study the ingredients of the Bethe-Yang description are the following: there is a single massless particle with fermionic statistics, the two-particle S-matrix is a constant scalar $S(k)=-1$, where $k$ is the relative momentum. The reflection matrix on the left-hand side can be read from (135), it is $R_{L}(k)=-1$; the reflection matrix on the right-hand side can be read from (136), it is

$$
R_{R}(k)=\frac{16 L h^{2}+\mathrm{i} k}{16 L h^{2}-\mathrm{i} k} .
$$

The transfer matrices for $N$-particle states are scalars:

$$
\begin{array}{r}
T_{i}\left(k_{1}, k_{2}, \ldots, k_{N}\right)=R_{L}\left(k_{i}\right) R_{R}\left(k_{i}\right) \prod_{j, j \neq k} S\left(k_{i}+k_{j}\right) \prod_{j, j \neq k} S\left(k_{i}-k_{j}\right)=-R_{R}\left(k_{i}\right), \\
i=1 \ldots N,
\end{array}
$$

where $k_{1}>k_{2}>\ldots k_{N} \neq 0$. This very simple form is the consequence of the simplicity of the S-matrix. The Bethe-Yang equations for the momenta $k_{1}, k_{2}, \ldots k_{N}$ of the $N$-particle states take the form

$$
\mathrm{e}^{2 \mathrm{i} k_{i} L} T_{i}\left(k_{1}, k_{2}, \ldots, k_{N}\right)=\mathrm{e}^{2 \mathrm{i} k_{i} L} \frac{\mathrm{i} k_{i}+16 L h^{2}}{\mathrm{i} k_{i}-16 L h^{2}}=1, \quad i=1 \ldots N
$$

The total energy of an $N$-particle state in the Bethe-Yang framework is $E=\sum_{i=1}^{N} k_{i}$. (150) can be rewritten as $k_{i} L \tan \left(k_{i} L\right)=16 L^{2} h^{2}, i=1 \ldots N$, which has the same form as (38). This means that the Bethe-Yang description reproduces the result of Section 2.2 for the spectrum exactly.

The reverse model is similar, one can read from (144) and (145) that

$$
R_{L}(k)=-1, \quad R_{R}(k)=\frac{1-\mathrm{i} k 16 L g}{1+\mathrm{i} k 16 L g},
$$

and the Bethe-Yang equations for the momenta can be written as

$$
k_{i} L \tan \left(k_{i} L\right)=\frac{-1}{16 g}, \quad i=1 \ldots N
$$

which has the same form as (62), i.e. the result of Section 3 for the spectrum is reproduced exactly. 


\section{Power series expansion of the energy levels}

The eigenvectors of $H_{0}$ suitable for Rayleigh-Schrödinger perturbation theory are those introduced in (21). Degenerate perturbation theory has to be used.

The nonzero matrix elements of $H_{I}$ in Section 2.2 are the following:

$$
\begin{aligned}
-\left\langle Q v\left|H_{I}\right| Q v\right\rangle & =\left\langle Q u\left|H_{I}\right| Q u\right\rangle=2 \\
\left\langle Q u\left|H_{I}\right| P v\right\rangle & =\left\langle P v\left|H_{I}\right| Q u\right\rangle=2 \sqrt{2}(-1)^{n+m}(-1)^{k L / \pi} \\
\left\langle Q v\left|H_{I}\right| P u\right\rangle & =\left\langle P u\left|H_{I}\right| Q v\right\rangle=-2 \sqrt{2}(-1)^{n+m}(-1)^{k L / \pi},
\end{aligned}
$$

where $P=a\left(k_{1}\right) a\left(k_{2}\right) \ldots a\left(k_{m}\right) a(k) a\left(k_{m+1}\right) \ldots a\left(k_{n}\right), Q=a\left(k_{1}\right) a\left(k_{2}\right) \ldots a\left(k_{n}\right)$.

We remark that certain perturbative calculations were also done in [39].

The eigenvalue of the state starting from $a\left(\frac{N_{1} \pi}{L}\right) a\left(\frac{N_{2} \pi}{L}\right) \ldots a\left(\frac{N_{r} \pi}{L}\right)|w\rangle$ at $h=0$ is

$$
\begin{aligned}
& E_{\left\{N_{1}, N_{2}, \ldots, N_{r}, w\right\}}(h)=\frac{\left(N_{1}+N_{2}+\cdots+N_{r}\right) \pi}{L}+2 \epsilon h \\
& +\left(\frac{16 L}{\pi}\left(\frac{1}{N_{1}}+\frac{1}{N_{2}}+\cdots+\frac{1}{N_{r}}\right)-\sum_{n=1}^{n_{m}} \frac{8 L}{n \pi}\right) h^{2}-\epsilon \sum_{n=1}^{n_{m}} \frac{32 L^{2}}{n^{2} \pi^{2}} h^{3}+\ldots,
\end{aligned}
$$

where $w$ stands for $v$ or $u, \epsilon=-1$ if $w=v, \epsilon=1$ if $w=u . n_{m}=n_{c}$ in the MT scheme ( $n_{c}$ is the truncation parameter introduced in Section 4.1), $n_{m}=n_{c}-\left(N_{1}+N_{2}+\cdots+N_{r}\right)$ in the TCSA scheme ( $n_{c}$ is the conformal truncation parameter), and $n_{m}=\infty$ in the non-truncated case.

In the non-truncated case the coefficient of $h^{2}$ is ultraviolet divergent and should be regularized. The TCSA and the mode truncation both provide a regularization.

We remark that the above formulae show that the truncated energy gaps converge to the non-truncated energy gaps as $1 / n_{c}$.

\section{References}

[1] Yurov V P and Zamolodchikov Al B, Truncated conformal space approach to scaling Lee-Yang model, 1990 Int. J. Mod. Phys. A 5 3221-46

[2] Feverati G, Ravanini F and Takács G, Nonlinear integral equation and finite volume spectrum of minimal models perturbed by Phi(1,3), 2000 Nucl. Phys. B 570 615-43 |hep-th/9909031|; Feverati G, Ravanini F and Takács G, Scaling functions in the odd charge sector of sineGordon / massive Thirring theory, 1998 Phys. Lett. B 444 442-50 [hep-th/9807160]; Feverati G, Ravanini F and Takács G, Nonlinear integral equation and finite volume spectrum of sineGordon theory, 1999 Nucl. Phys. B 540 543-86 [hep-th/9805117]; Feverati G, Ravanini F and Takács G, Truncated conformal space at $c=1$, nonlinear integral equation and quantization rules for multi-soliton states, 1998 Phys. Lett. B 430 264-73 [hep-th/9803104] 
[3] Pozsgay B and Takács G, Characterization of resonances using finite size effects, 2006 Nucl. Phys. B 748 485-523 |hep-th/0604022]

[4] Bajnok Z, Palla L, Takács G and Wágner F, A nonperturbative study of the two-frequency sine-Gordon model, 2000 Nucl. Phys. B 601 503-38 [hep-th/0008066

[5] Tóth G Zs, A non-perturbative study of phase transitions in the multi-frequency sine-Gordon model, 2004 J. Phys. A 37 9631-50 [hep-th/0406139]

[6] Kormos M, Boundary renormalisation group flows of unitary superconformal minimal models, 2006 Nucl. Phys. B $744358-79$ hep-th/0512085

[7] Recknagel A, Roggenkamp D and Schomerus V, On Relevant Boundary Perturbations of Unitary Minimal Models, 2000 Nucl. Phys. B 588 552-64 [hep-th/0003110|

[8] Dorey P, Pocklington A, Tateo R and Watts G M T, TBA and TCSA with Boundaries and Excited States, 1998 Nucl. Phys. B 525 641-63 hep-th/9712197

[9] Graham K, Runkel I and Watts G M T, Renormalisation Group Flows of Boundary Theories, 4th Annual European TMR Conference on Integrability, Nonperturbative Effects and Symmetry in Quantum Field Theory, Paris, France, 7-13 Sep 2000 hep-th/0010082

[10] Graham K, Runkel I and Watts G M T, Boundary Renormalisation Group Flows of Minimal Models, Non-perturbative QFT methods and their applications: proc. of the 24th Johns Hopkins Workshop on Current Problems in Particle Theory, Budapest, Hungary, ed Z Horvath and L Palla (Singapore: World Scientific, 2001) pp 95-113

[11] Dorey P, Pillin M, Pocklington A, Runkel I, Tateo R and Watts G M T, Finite Size Effects in Perturbed Boundary Conformal Field Theories, 4th Annual European TMR Conference on Integrability, Nonperturbative Effects and Symmetry in Quantum Field Theory, Paris, France, 7-13 Sep 2000 hep-th/0010278

[12] Chatterjee R, Exact Partition Function and Boundary State of 2-D Massive Ising Field Theory with Boundary Magnetic Field, 1996 Nucl. Phys. B 468 439-60 hep-th/9509071]

[13] Graham K and Watts G M T, Defect lines and boundary flows, 2004 JHEP 0404019 |hep-th/0306167|

[14] Fredenhagen S, Organizing Boundary RG Flows, 2003 Nucl. Phys. B 660, 436-72 hep-th/0301229

[15] Lesage F, Saleur H and Simonetti P, Boundary flows in minimal models, 1998 Phys. Lett. B 427 85-92 [hep-th/9802061|

[16] Cappelli A, D'Appollonio G and Zabzine M, Landau-Ginzburg description of boundary critical phenomena in two-dimensions, 2004 JHEP 0404010 hep-th/0312296

[17] Klassen T R and Melzer E, Spectral flow between conformal field theories in $1+1$ dimensions, 1992 Nucl. Phys. B 370 511-50 
[18] Cardy J L, Lässig M and Mussardo G, The scaling region of the tricritical Ising model in two-dimensions, 1991 Nucl. Phys. B 348 591-618

[19] Watts G M T, 2004, private communication

[20] Feverati G, Graham K, Pearce P A, Tóth G Zs and Watts G, A Renormalisation group for TCSA, talk presented by G. Watts at the workshop "Integrable Models and Applications: from Strings to Condensed Matter", Santiago de Compostela, Spain, 12-16 September 2005 hep-th/0612203

[21] Cardy J, Boundary Conditions, Fusion Rules And The Verlinde Formula, 1989 Nucl. Phys. B 324581

[22] Cardy J and Lewellen D, Bulk and boundary operators in conformal field theory, 1991 Phys. Lett. B 259 274-78

[23] Ghoshal S and Zamolodchikov A, Boundary S matrix and boundary state in two-dimensional integrable quantum field theory, 1994 Int. J. Mod. Phys. A 9 3841-86, Erratum-ibid. A 94353 hep-th/9306002

[24] Affleck I and Ludwig A W W, Universal Noninteger "Ground-State Degeneracy" in Critical Quantum Systems, 1991 Phys. Rev. Lett. 67 161-4

[25] Feverati G, Graham K, Pearce P A and Watts G M T, to appear

[26] Chatterjee R, Exact Partition Function and Boundary State of Critical Ising Model with Boundary Magnetic Field, 1995 Mod. Phys. Lett. A 10 973-84 |hep-th/9412169

[27] Chatterjee R and Zamolodchikov A, Local Magnetization in Critical Ising Model with Boundary Magnetic Field, 1993 hep-th/9311165

[28] LeClair A, Mussardo G, Saleur H and Skorik S, Boundary energy and boundary states in integrable quantum field theories, 1995 Nucl. Phys. B 453 581-618 [hep-th/9503227|

[29] Konechny A, Ising model with a boundary magnetic field: an example of a boundary flow, 2004 JHEP 0412058 [hep-th/0410210]

[30] Konik R, LeClair A and Mussardo G, On Ising correlation functions with boundary magnetic field, 1996 Int. J. Mod. Phys. A 11 2765-82 [hep-th/9508099]

[31] Di Francesco P, Mathieu P and Sénéchal D, Conformal Field Theory, Graduate Texts in Contemporary Physics, Springer-Verlag

[32] Ginsparg P, Applied Conformal Field Theory, 1989 Fields, Strings and Critical Phenomena (Les Houches, Session XLIX, 1988) ed E Brézin and J Zinn-Justen (Elsevier) [hep-th/9108028]

[33] Tóth G Zs and Watts G M T, to appear

[34] Zamolodchikov A B, Thermodynamic Bethe Ansatz In Relativistic Models. Scaling Three State Potts And Lee-Yang Models, 1990 Nucl. Phys. B 342 695-720 
[35] Klassen T R and Melzer E, Kinks in Finite Volume, 1992 Nucl. Phys. B 382 441-85 hep-th/9202034

[36] Bajnok Z, Palla L, Takács G and Wágner F, The k-folded sine-Gordon model in finite volume, 2000 Nucl. Phys. B 587 585-618 hep-th/0004181]

[37] Fendley P and Saleur H, Deriving boundary S matrices, 1994 Nucl. Phys. B 428 681-93 hep-th/9402045

[38] Bajnok Z, Palla L and Takács G, Boundary states and finite size effects in sine-Gordon model with Neumann boundary condition, 2001 Nucl. Phys. B 614 405-48 [hep-th/0106069]

[39] Caux J S, Saleur H and Siano F, The two-boundary sine-Gordon model, 2003 Nucl. Phys. B 672 411-61 cond-mat/0306328 